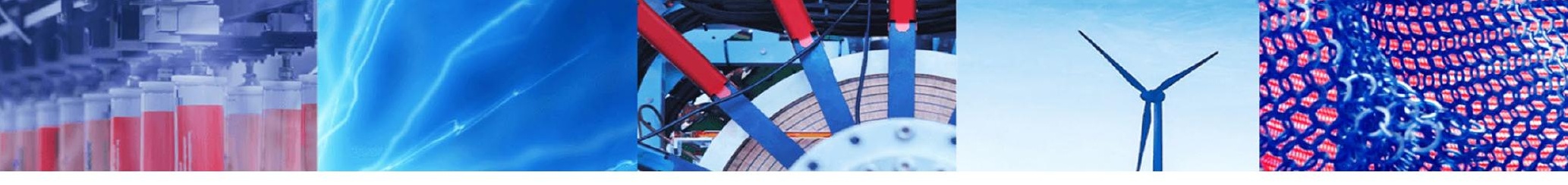

Research Article

\title{
A low latency MAC protocol for underwater sensor networks considering bi-directional communication in multi-hop and multi-flow scenarios
}

\author{
Jenifar Rahman ${ }^{1}$ Md. Zahirul Islam ${ }^{1} \cdot$ William Luke Lambert ${ }^{2}$ Md. Saiful Islam ${ }^{1} \cdot$ Mohammad Shah Alam $^{1,2}$ (I)
}

Received: 30 May 2019 / Accepted: 24 October 2019 / Published online: 11 November 2019

(c) Springer Nature Switzerland AG 2019

\begin{abstract}
Underwater Acoustic Sensor Network (UW-ASN) is a remote system comprising of spatially disseminated independent gadgets utilizing sensors to screen physical or natural conditions underwater. Regular MAC protocol is not adequate in underwater acoustic sensor systems (UW-ASN) due to high error rate and long propagation delay for the general attributes of the physical media. In this research work, a Bidirectional Multi-Flow MAC protocol (BMF-MAC) for UW-ASN is proposed in order to manage multi-hop and multi-stream data flows so different flows of data transmission continue at the same time while modifying by fluctuating traffic state. The proposed protocol provisions structure of multi-hop streams, contrary to other underwater MAC protocols, by considering each pending packets in directing layer support. All stream setup request from neighbors while setting up a stream. So as to complete the execution investigation of the proposed BMF-MAC protocol, an experimental model is designed, which incorporates the condition of energy utilization, throughput, end to end delay and frame error likelihood. Comparative performance analysis has been done between the proposed protocol and the contemporary Cascading Multi-jump Reservation and Transmission protocol and performance is analyzed in terms of throughput, end-to-end delay, and energy utilization.
\end{abstract}

Keywords Underwater acoustic sensor networks (UW-ASN) - Long propagation delay · MAC · Bi-directional communication · CMRT

\section{Introduction}

Different applications for the underwater vehicles is the developing innovation for Underwater Acoustic Sensor Network (UW-ASN). Ecological observing, asset examination, disaster recovery and military observation are a portion of the widely utilized applications mentioned in [1]. Such a system regularly comprises of countless nodes that arrange themselves into a multi-hop organize. Every node has at least one sensor, installed processors and acoustic modem, and is typically battery worked. Commonly, these nodes organize to perform collective observing assignments over a foreordained zone.

Medium Access Control (MAC) protocol in underwater sensor networks has an important role to enable the successful operation of the network. If two interfering nodes are unable to transmit information at a similar time, collision can be evaded at the same time which is the basic job of the protocol. The proposed protocols for UWSNs by and large can be ordered into three kinds: ALOHA-based, time division multiple accesses (TDMA)-based and carrier sense multiple access (CSMA)-based in recent year.

$\triangle$ Mohammad Shah Alam, msalam@tntech.edu; Jenifar Rahman, jenifar_shithi@yahoo.com; Md. Zahirul Islam, zahir375@gmail.com; William Luke Lambert, wllambert42@students.tntech.edu; Md. Saiful Islam, mdsaifulislam@iict.buet.ac.bd| ${ }^{1}$ IICT, Bangladesh University of Engineering and Technology, Dhaka, Bangladesh. ${ }^{2}$ Department of Computer Science, Tennessee Technological University, Cookeville, USA. 
As the area of ocean environment for sensing are large, the sensor nodes become costly. In this manner, the primary requirement is to enrich the utilization of mobile sensor and deploy sparse network. As the battery capacity of underwater sensors are limited, they may face corrosion problem. Furthermore, due to the unavailability of of the solar power in underwater, battery of the sensor node cannot be recharged in typical way [2].The fundamental differences between terrestrial WSN and UW-ASN are discussed in the following. It is mentioned in [3], in order to transmit packet the radio frequency (RF) electromagnetic signal along with the speed of light $\left(3 \times 10^{8} \mathrm{~m} / \mathrm{s}\right)$ is used by the network where the propagation time is avoided. Due to the usage of UW-ASN acoustic wave $(1500 \mathrm{~m} / \mathrm{s})$ as carrier for communication "space-time uncertainty" come up where propagation time is an important parameter will be longer than typical RF electromagnetic signals. As the transmitting power is 100 times more powerful than receiving power in acoustic links in [4], consumption of transmitting power in UW-ASN becomes expensive. Due to the fact, the terrestrial protocols are hopelessly ineffective to use in UW-ASN.

Moreover, in underwater environment, the bandwidth is restricted by the attributes, for example, path loss, noise, multi-path, high delay variance, and Doppler-spread in [5]. In acoustic system, due to the condition of physical media, long proliferation delay and high packet error rate is observed which is mentioned in [6]. As a result, the wide assortment of MAC conventions formerly proposed for remote terrestrial systems don't perform well in underwater because of the previously mentioned uniqueness of underwater systems.

A preliminary version of this work was presented in [7]. The rest of the paper is organized as follows: Sect. 2 briefly describes recent contributions. In Sect. 3, the architecture of UW-ASN and communication model is described. The proposed BMF-MAC protocol is illustrated in Sect. 4. The state transition diagram of sensor node illustrated in Sect. 5. In Sect. 6, the experimental setup and results are discussed. The paper is been concluded in Sect. 7.

\section{Related work}

In underwater environment, the design of handshakingbased MAC protocol is a complex work due to the abnormal nature of acoustic signal which has a long propagation delay. A number of works have been proposed to reduce handshaking significantly [8-13]. A few protocols have enhanced the channel use by sending various packets without a moment's delay in a packet-train form. In [8], a receiver-initiated MAC protocol is coined as Receiver-Initiated Packet Train (RIPT) protocol. Helper node is coined in Four-way handshaking protocol (readyto-receive (RTR)/SIZE/ORDER/DATA), where packets from distinct neighbors arrive at the collector. In the underwater environment, it takes a lot time to receive the main packet train at the receiver side if four-way handshaking is used when neighbors send various data in RIPT.

A hybrid MAC protocol is coined and used by both sender and receiver in [9], titled as the Reverse Opportunistic Packet Appending (ROPA) protocol. By preferentially transmitting the data packets, sender coordinate different neighbors in this protocol which enhance the utilization of channel. If more control packet is exchanged, this protocol may faces more collision.

Furthermore, handshake-sharing approach is introduced in $[9,10]$ has allowed numerous nodes to partake in a typical handshake. Furthermore, some of the works adopted a sender-initiated approach in order to provide solution while others adopted receiver-initiated approach to provide solution. In this research work, we focus on the study of the handshaking approach to improve performance of the sensor networks.

Furthermore, to decrease sudden impacts at the recipient because of shrouded neighbors, Hybrid sender-and beneficiary started (HSR) convention [10] empowers all hubs to keep up a vital good ways from concealed hub incited crashes as demonstrated by a luxuriously decided holding up time. The convention proposes a method for sharing the handshakes of control parcels among various hubs. Handshake-sharing can be practiced by inviting neighbors to join the present handshake and by empowering them to send their information parcels without requiring extra handshakes. In MACA-MN [11], information transmission during communicating with the neighbors, fruitful handshake by the sender is very much essential.

The different access impact evasion (MACA) convention utilizes the solicitation to-send (RTS)/clear-to-send (CTS) handshake for saving the common channel which is a well known earthbound handshake-based MAC convention [12]. For completing MACA in a submerged situation, MACA for submerged (MACA-U) was proposed which can reconsider the state change principles considering the long spread deferral [13]. Be that as it may, because of the long proliferation delay in the submerged acoustic channel, the basic RTS/CTS trade can not completely address the covered up hub issue. In request to address triple shrouded terminal issue for multi-channel transmission for submerged sensor systems, CUMAC-CAM is proposed in $[14,15]$. Additionally, the necessity of expanded time for trading the control bundle makes the exhibition of the convention seriously compelled. Moreover, a spatial shamefulness issue emerges because of the long 
proliferation delay in submerged condition represented in [16].

MACA-U with bundle trains (MACA-UPT) was likewise exhibited in [17]. MACA-UPT is gotten from MACA-U, of course, really a sender transmits distinctive data packages in a single handshake in the past.

Slotted-FAMA is a main MAC convention which combine both profession detecting and handshaking framework for collision [18]. In this convention, parcels are transmitted at the beginning of an opening whose length is proportional to the greatest proliferation delay. In any case, the throughput execution is on a very basic level lessened by the unnecessary length of the space, anyway the convention can check effects brought about by concealed hubs.

In [19], bidirectional concurrent MAC (BiC-MAC) protocol is proposed to transmit data packets to a senderreceiver pair simultaneously for each successful handshake by improving channel utilization. The protocol adopts a packet bursting method that allows the sender and receiver node pair to exchange multiple rounds of bidirectional packet transmissions. Thus, the entire set of data packets is transmitted over several discontinuous packet bursts. Therefore, single sender and receiver is considered for bidirectional data transmission. However, multi-flow scenario is not considered.

In order to make receiver receives information without collision, the transmitter transmit data packet in train way which is the plan of the protocol. However, the protocol is excessively entangled to viably enhance network throughput and need excessively control packets which will impact the system performance.

Also, holding multi-hop channels without a moment's delay in series way, the Cascading multi-hop reservation and transmission (CMRT) protocol mentioned in [19] where data packets are sent to the destination by transferring data packets dynamically. Besides, CMRT receives a strategy dependent on packet train show so as to enhance usage of channel.

Even though various authors have submitted quality routing protocol papers and study papers in various territories of UWSNs, motionless possibility of the routing protocol introduced in this article is recognized from the current works in numerous perspectives [20-23]. Comparative analysis of routing algorithm for underwater wireless sensor network is been analyzed in [24].

\section{Architecture of underwater acoustic sensor networks}

The underwater sensor organize topology is an open research issue that needs increasingly logical and simulative examination from the exploration network. The correspondence design of underwater acoustic sensor systems are depicted in this segment. Here we depict the reference designs which are utilized as a reason for dialog of the difficulties related with underwater acoustic sensor networks [25]. Static UW-ASNs are set up by sensor nodes that are tied down to the base of the sea. There normal applications are natural checking, or observing of underwater plates in tectonics [26].

Figure 1 shows a reference design for underwater systems. Here, we watch a gathering of sensor nodes are moored to the base of the sea with profound sea stays. Underwater sensor nodes are interconnected to at least one underwater sinks (UW-sinks) with the assistance of wireless acoustic connections. These are network gadgets accountable for transferring information from the sea base system to a surface station. By and large, UW-sinks are furnished with two acoustic transceivers called a vertical and an horizontal transceiver. UW-sink utilizes the horizontal transceiver for communicating with the sensor nodes. They can send directions and setup information to the sensors (UW-sink to sensors), gather observed information (sensors to UW-sink). Besides, UW sinks utilize the vertical connection also. They can hand-off information to a surface station. Vertical transceivers ought to be long range transceivers for profound water applications as the sea can be as profound as $10 \mathrm{~km}$. The surface station which is able to deal with various parallel correspondences with the sent UW-sinks is outfitted with an acoustic transceiver. Besides, it is blessed with a long range RF and additionally satellite transmitter to speak with the Onshore sink (OSsink) or to a surface sink (s-sink).

By methods for direct connections or through multihop ways, sensors are associated with UW-sinks. Every sensor straightforwardly sends the assembled information to the chose UW-sink in the primary case. In spite of the fact that this is the most effortless approach to arrange sensors, it is less energy efficient. Since, the sink might be a long way from the hub and the power important to transmit may rot with forces more prominent than two of the separation. In addition, because of expanded acoustic obstruction brought about by high transmission control, direct connections are exceptionally prone to decrease the system throughput. As in earthbound sensor networks [27], for multi-bounce ways condition, the information achieved by a source sensor is sent by middle of the road sensors until it achieves the UW-sink. This causes more vitality investment funds and builds arrange limit. In any case, this expands the multifaceted nature of the directing usefulness. Each system gadget more often than not participates in a community procedure. Their key duty is to diffuse topology data to such an extent that proficient and circle free directing choices can occur at each moderate hub. This can 
Fig. 1 Architecture for underwater sensor networks

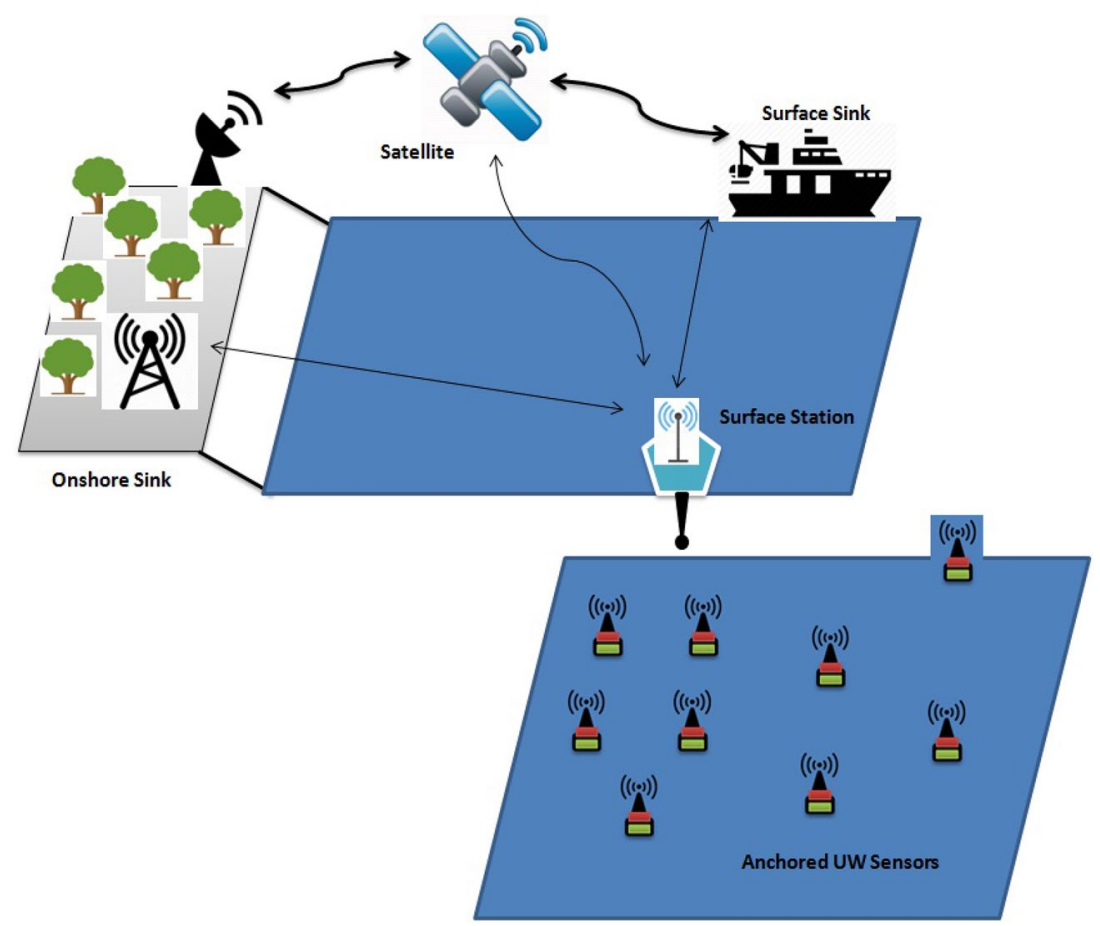

be accomplished by the component which comprises of flagging and calculation. From above discourse we can presume that, vitality and limit are valuable assets in submerged situations. The basic objective in UW-ASNs is to convey occasion includes by abusing multi-jump ways and limiting the flagging overhead important to build underwater ways in the meantime.

\section{Proposed MAC protocol}

The proposed BMF-MAC protocol is aimed at improving the performance of the existing CMRT protocol [19]. At first, the operation cycles of the proposed MAC protocol along with its control frame structure is illustrated. Multihop multi-flow data forwarding with reverse packet method is outlined here as well. Transition diagram and algorithm for data transmission technique for both sender and relay node are also laid outed.

\subsection{Network mode}

In our proposed protocol flow is set in such a manner that, multiple flows can be constructed from single node, thus a node can transmit multiple packets over multiple flows. Furthermore, a sender can sends different packets to different multiple destination nodes. From intermediate node of the flow may add additional final destinations.

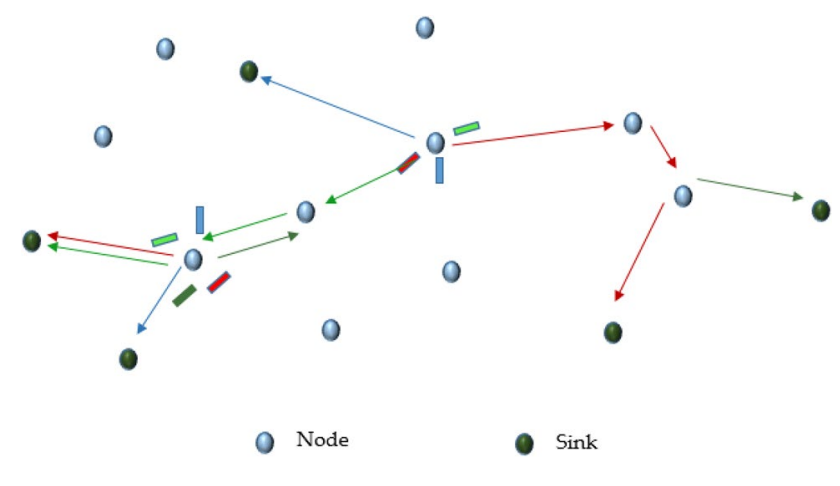

Fig. 2 Network model

The network model is considered with multiple sinks. The network model is illustrated in Fig. 2.

\subsection{Definition of states}

Among fourteen dissimilar conditions, six conditions are coined in similar way of CMRT protocol and four new states are coined in the proposed BMF-MAC protocol to take care of data transmission by the reverse flow direction. Figure 3 illustrates the states of the protocol.

In Wait_Resp state, receiver sends request control packet (RTS) and sender waits for it. The sender stays in the Wait_Resp state till to receive a response control packet after sending request control packet. If the sender node does not get a response control packet during the tenure 
Fig. 3 States of BMF-MAC protocol

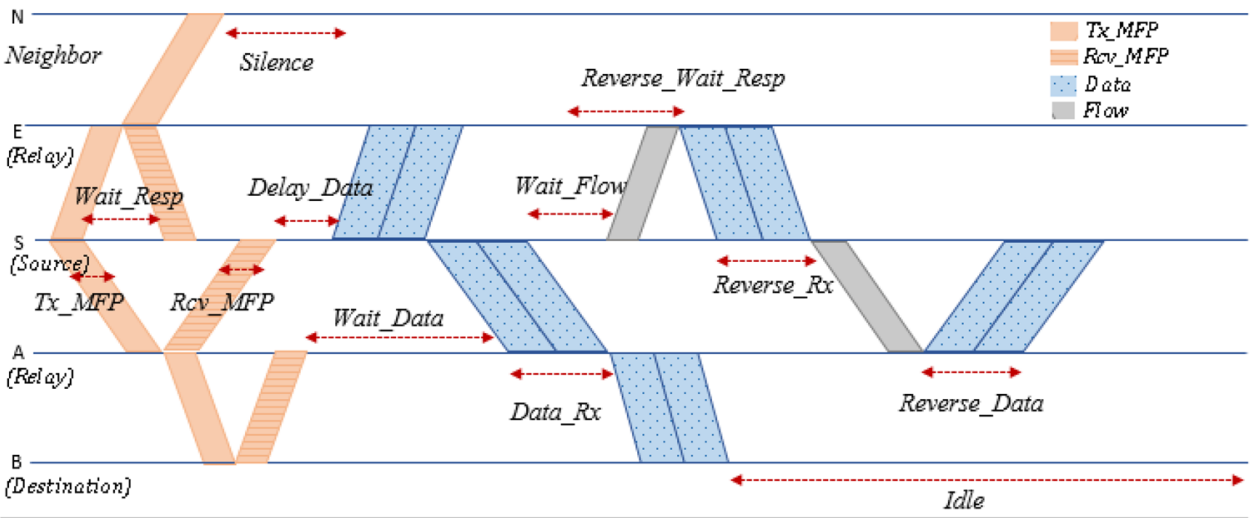

of Wait_Resp state, it will move on a new state coined Idle state.

In order to bypass the tentative collisions generated by the hidden nodes, data transmission process is delayed by the sender in named as Delay_Data state. The sender node moves into the Delay_Data state and waits till to begin sending data packets after getting the response control packet sent by the receiver node.

Moreover in the Wait_Data state receiver nodes await till to get the data packets is sent by the sender. The receiver node moves into the Wait_Data state straightly and waits till to begin receiving data packets after getting the response control packet.

In Data_Rx state, data packets are received by the receiver.

At the time of exchanging the control packets because of channel reservation is overheard by the neighbor nodes keep silent in the Silence state.Due to their inactivity no collisions occur.

When neighbor nodes overhear the control packets which is applied to reserve the channel of other nodes moves into silence state and stay there till the channel turn into free from reservation. At the receiver side this state confirms that after finishing the data packet reception, other transmission from neighbor nodes initiate.

The Idle state is a state where a node has no activity to do.

A state coined $T x_{-}$MFP is familiarized where MFP packet is entreated to send to relay node. Besides another state is defined as RCv_MFP where MFP from receiver is confirmed by a node.

In a state where a receiver anticipates for the forward data transmission which is about to finish over a flow is named as Wait_Flow state. After transferring bunch data packets, receiver moves to the Wait_Flow state unswervingly and waits there until starting the CTS transmission because of reverse data transmission.

In the Reverse_Wait_Resp state receiver sends CTS packet as response and sender waits for it. If a sender

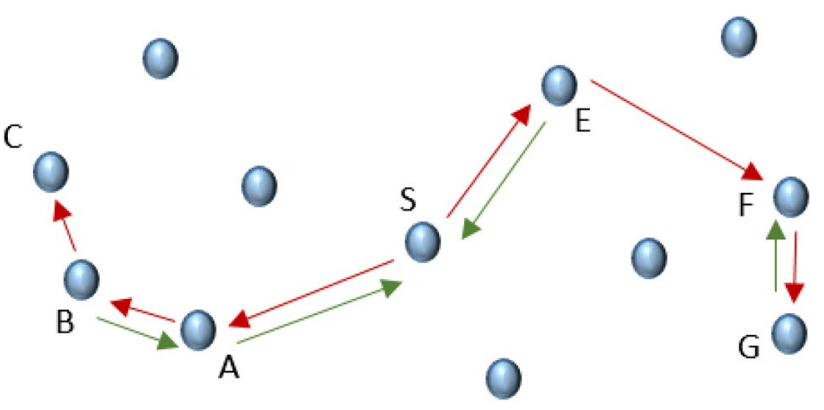

$\uparrow$ Regular Flow $\uparrow$ Reverse Flow

Fig. 4 Topology

node observes the set state of reverse flag after finishing its data transmission in forward direction, it will wait to begin data transmission in reverse direction of the same flow.

In order to evade potential collisions because of bidirectional data transmission, sender node makes delay the transmission of data in Reverse_Data state. In the interim, a CTS is sent by the receiver to the sender node and after getting it sender enters into Reverse_Data state. Sender stays in the same state up to completion of reverse data packet transmission.

When a receiver accepts data packets from reverse flow, the state is coined as Reverse_Rx state.

In order to handle missing control packets during data transmission, $T x \_$Retry and Wait_Retry states are introduced. In $T x \_$Retry state, sender node will transmit extra control packet which is coined as Retry packet (RP) when corresponding receiver node does not get any data packet. Besides, if any sender node does not receive authorize MFP, will wait for RP from receiver in Wait_Retry state. 


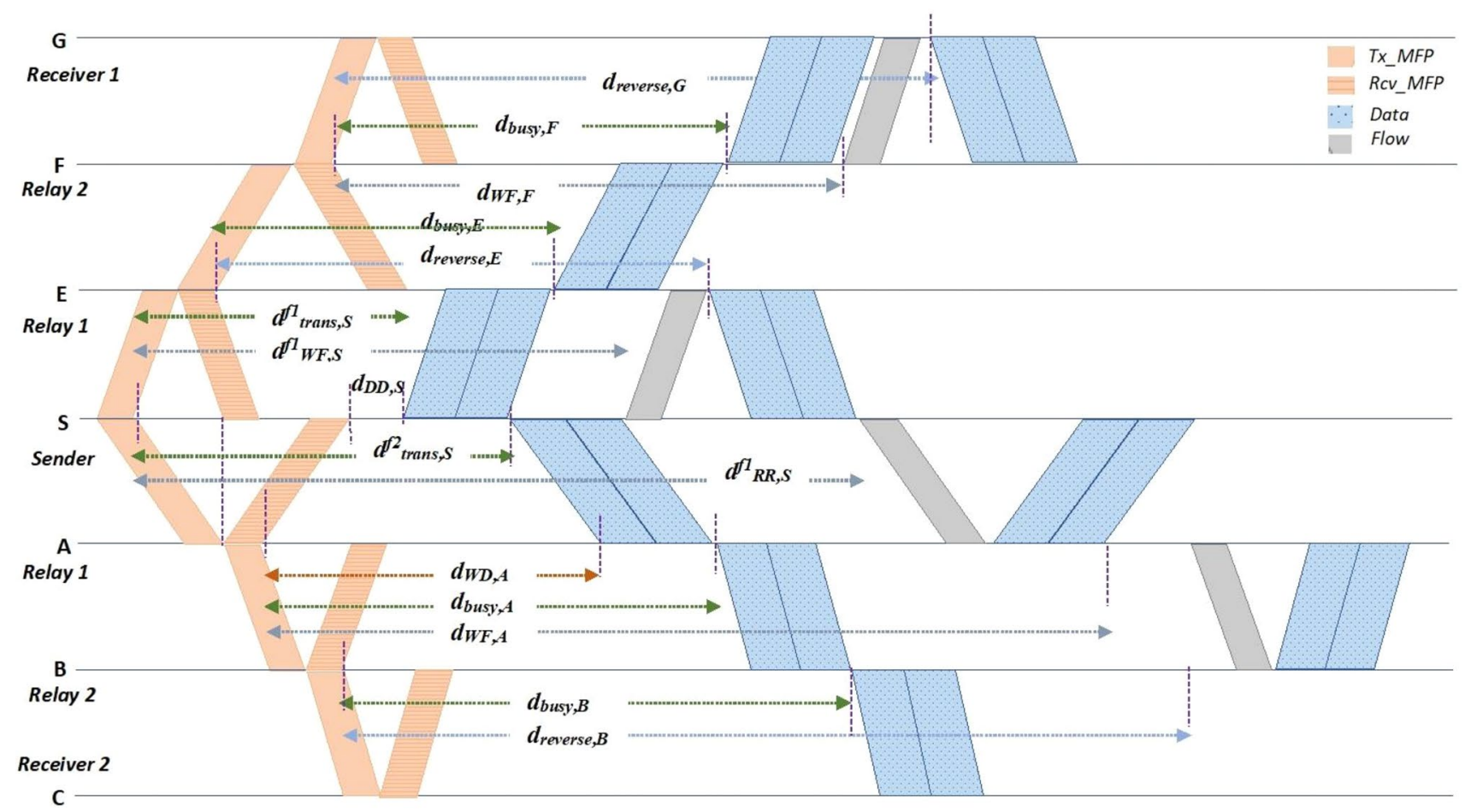

Fig. 5 Illustration of working principal bi-directional multi-flow MAC protocol

\subsection{Illustration of protocol}

The topology and the activity of the proposed protocol is illustrated in Figs. 4 and 5 correspondingly. In Fig. 4, sender node is denoted as node $S$. Assume node $S$ contains clump packets for final node $C$ and $G$. Here, relay nodes $E$ and $F$ remain between source $S$ and destination $G$ in one flow direction. On the other hand, between source $S$ and destination $C$ relay nodes $A$ and $B$ remain in other stream direction. Consequently, node $S$ transmit packets to receiver node $C$ through node $A$, and $B$ similarly receiver node $G$ through node $E$, and $F$ individually.

In the proposed protocol, a new control packet is introduced at the time the source node $S$ start to transmit MFP to relay node $E$ and $A$ accordingly so that relaying procedure of multi-flow is recognized. Sender $\mathrm{S}$ go through the Wait_Resp state after sending MFP as like the CMRT protocol mentioned in [19]. Handshaking process between sender node $S$, destination node $E$ and $A$ by transmitting MFP to node $E$ at first then to node $A$. The priority of getting message depends on node distance.

A MFP bundle is sent to the transfer hub $F$ from the main goal, hub $E$ in the forward way where hub $S$ catches MFP in the turn around way. So also a MFP parcel is sent to transfer hub $B$ from the subsequent goal, hub $A n$ in the forward way where hub S catches MFP in invert way. Because of the detachment between hubs the proliferation deferral happens and two streams can occur simultaneously in our proposed convention. Consequent to handing-off MFP, sender hub S enters the state Delay_Data. On the other hand, both transferring hub An and E go in the state Wait_Data. Subsequent to tolerating S's MFP, hub A plays out unclear advance from hub $E$.

As the hub $\mathrm{E}$ is accepted as first address, it has the benefit to plan for the two information transmission i.e forward and invert. The hub $\mathrm{S}$ retransmits the information parcel to E after the time interim of Delay_Data $\left(D_{D D, S}^{f 1}\right.$, if the affirmation MFP isn't gotten before by hub $S$ than next jump $E$. In this way, $D_{D D, S}^{f 1}$ means the measure of time deferral of hub $S$ to transmit information from first stream. The arrangement of information parcels are transmitted by the hand-off hub $\mathrm{E}$ to the a while later transfer hub $\mathrm{F}$ uninterruptedly without getting whenever break among bundles. Individually, the arrangement of information bundles likewise handedoff by the hub $\mathrm{F}$ without having interval for the reason that the multi-jump channels are kept guided while in transit to the goal hubs.

The source hub $S$ abstains from transmitting information for the time Delay_Data $\left(D_{D D, S}^{f 1}+d_{\text {data }}\right)$ so as to sidestep the potential impacts which will emerge from transmission 


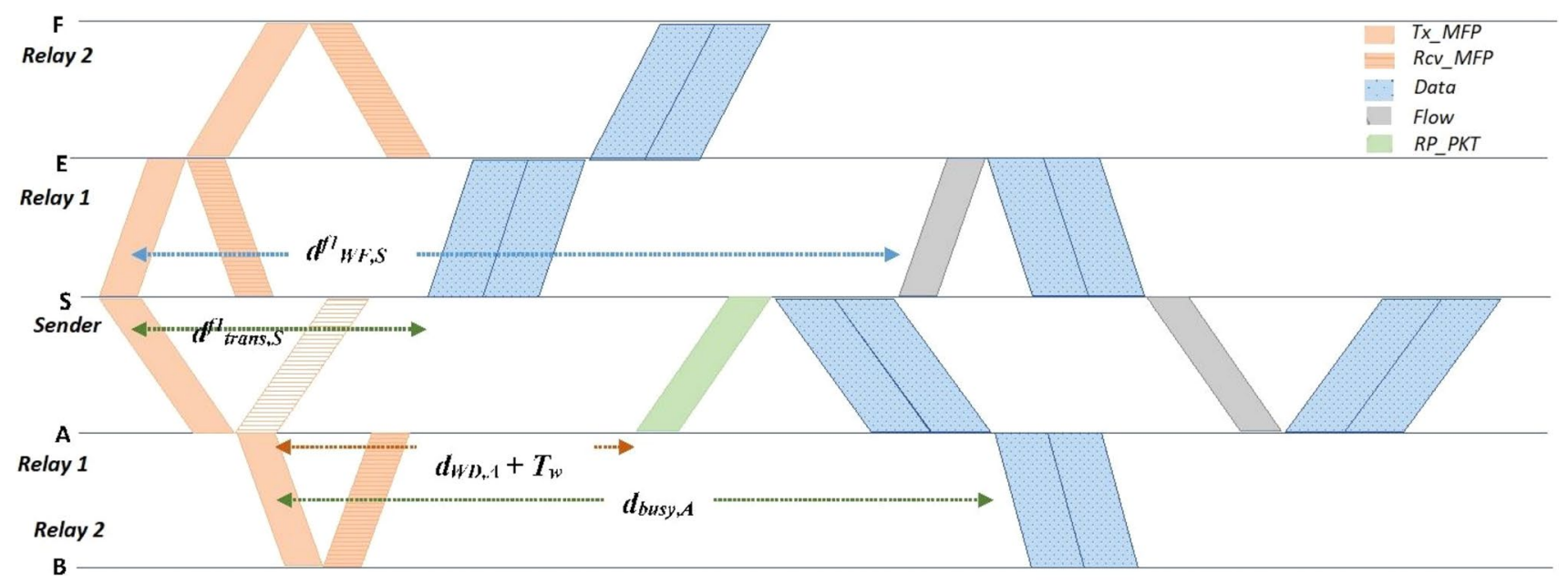

Fig. 6 Situation 1: sender slips a validation

of information over different streams. The first and second stream pursue the comparative information conveyance process. Each bounce pursue the comparable information conveying procedure up to definite goal $\mathrm{C}$ is come to. In this manner, information transferring is a pipeline procedure. In the proposed convention information from two individual stream can be conveyed simultaneously.

\subsubsection{Sending of data implementing the reverse packet technique}

By utilizing antithesis multi-stream packet method where the control packet MFP acquires a data sending method by sending dissimilar information packets from comparable stream in reverse direction. The exploitation of the channel will be enhanced from this time.

Figure 6 demonstrates the use of the invert packet system in the BMF protocol. Here, two surges of information transmissions at the same time happened. The transmission of information switching happens after fruitful information transmission from both source $S$ to $G$ and $S$ to $C$. While transmitting MFP, if a node comprises a packet from reverse comparative stream, sets switch banner to 1. By seeing the received reverse packet a node sends to sender node a CTS packet and sender transmits another packet to the final node.

There are some turn around parcels from hub $E$ to $S$ and hub $G$ to $F$ stay for the essential stream out of $S$ to $G$. In addition hub $S$ sets switch standard to 1 while sending MFP from hub $S$ to $E$. Likewise, hub F transmits MFP to hub G. Hub $S$ moves in Wait Flow state and sends CTS to $E$ after sending of data parcels from hub $S$ to $E$ and $S$ to $A$. Afterward, hub E transmit data bundle to $S$. After finish forward data transmission from hub $F$ to $G$, hub F sends CTS to $G$ because of no transmission jumps out at one bounce separation of hub F. At long last switch data bundle is sent from hub $G$ to $F$ right away.

Receiver node $S$ expected several packets from node $A$, while node $B$ has group packet for the target node $A$. After finishing the forward and reverse sending of node $E$, the node $A$ begins reverse information sending as the node $A$ is set as secondary goal. In order to send information from node $B$ to $A$, similar process is been followed. In Algorithm 1 the reverse waiting time calculation has been illustrated.

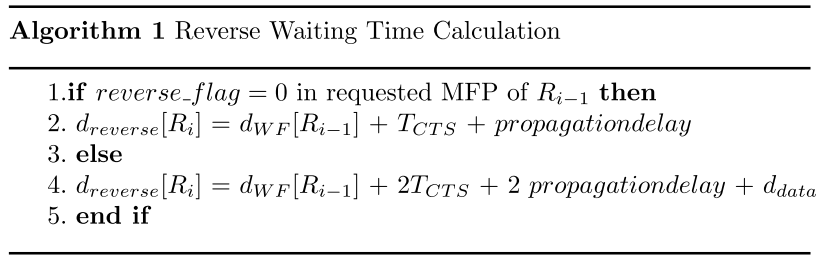

\subsubsection{Sending of data implementing request packet technique}

The Algorithm 2 and 3 explain the retry technique of handling any missing MFP packets.

Scenario 1: Sender failures a validation: Assume in Fig. 6 , the entreated MFP of node A is not received by the sender node $S$ which is coined as receive confirmation MFP. In Wait_Data state, $d_{W D, A}$ is denoted as waiting period of node $A$. The validation MFP does not acknowledged by node $S$ which is presumed by node $A$, when receiver $A$ does not receive any data after $d_{W D, A}+T_{w}\left(T_{w}\right.$ is a short waiting time) period. Consequently, sender 


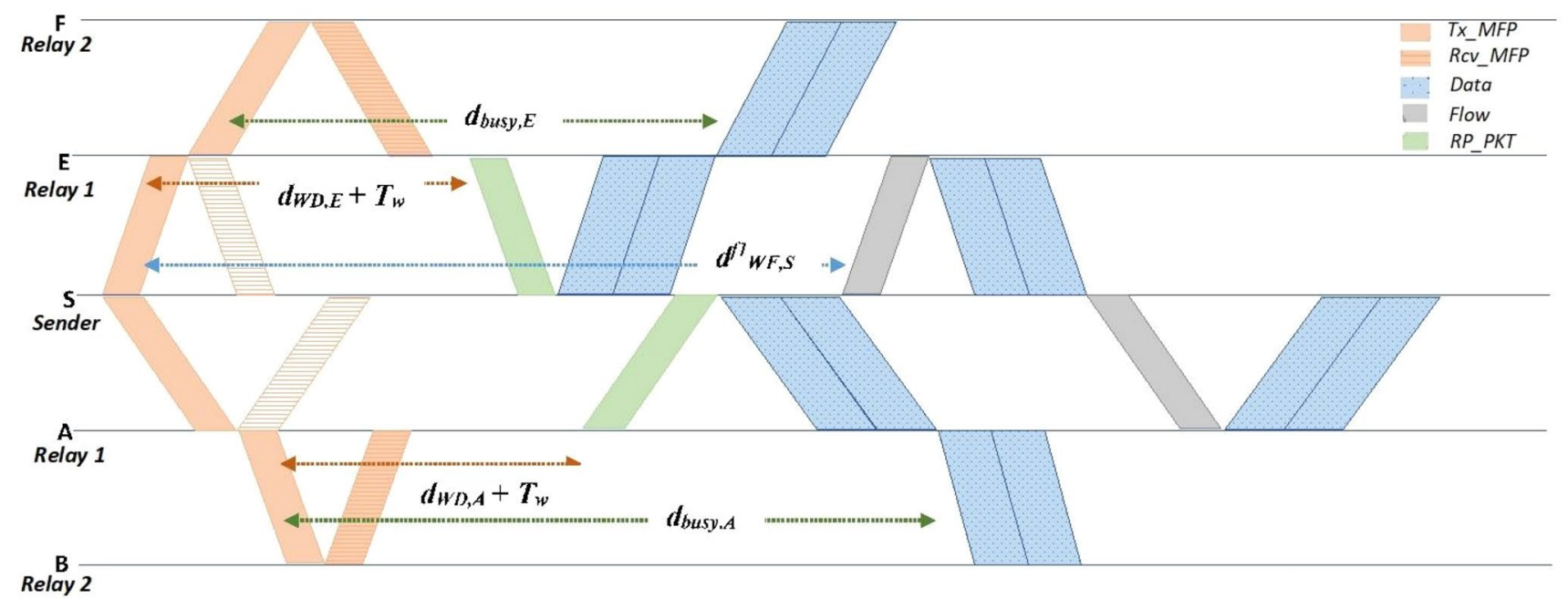

Fig. 7 Situation 2: sender slips all validation

node $S$ initiates to send data packets after sending RP packet to $A$ for second flow.

Scenario 2: Sender misses all validation: The sender hub $S$ doesn't get affirmation demand in the second situation for the two hubs $E$ and $A$ which is shown in Fig. 7. Hub E sends RP bundle when collector E doesn't get information from sender $S$ after $d_{W D, E}+T_{w}$ timeframe, and the sender hub $S$ starts to send information parcels immediately for first stream to hub E. Correspondingly, when recipient $A$ doesn't get information bundle from hub $S$ after $d_{W D, A}+T_{w}$ length time, hub A sends RP parcel and the sender hub $S$ starts to send information parcels for second stream to hub A speedily.

Scenario 3: Transitional node misses a validation: Let, the confirmation MFP which is sent by node $F$, is not received by node $E$ because of the packet collision. Due to not getting data packet from relay node $E$ by node $F$, start transmitting RP packet so that node $E$ starts relaying data packets to node $F$ instantaneously.

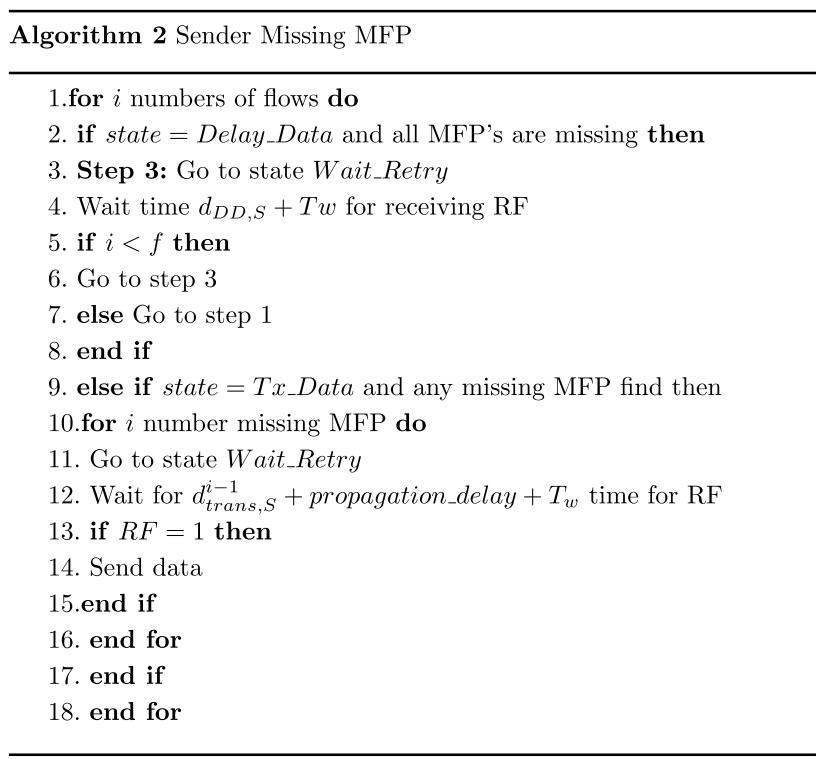


Fig. 8 Illustration of state evolution diagram of a sender node in proposed protocol
Fig. 9 Illustration of state evolution diagram of a relay node in proposed protocol
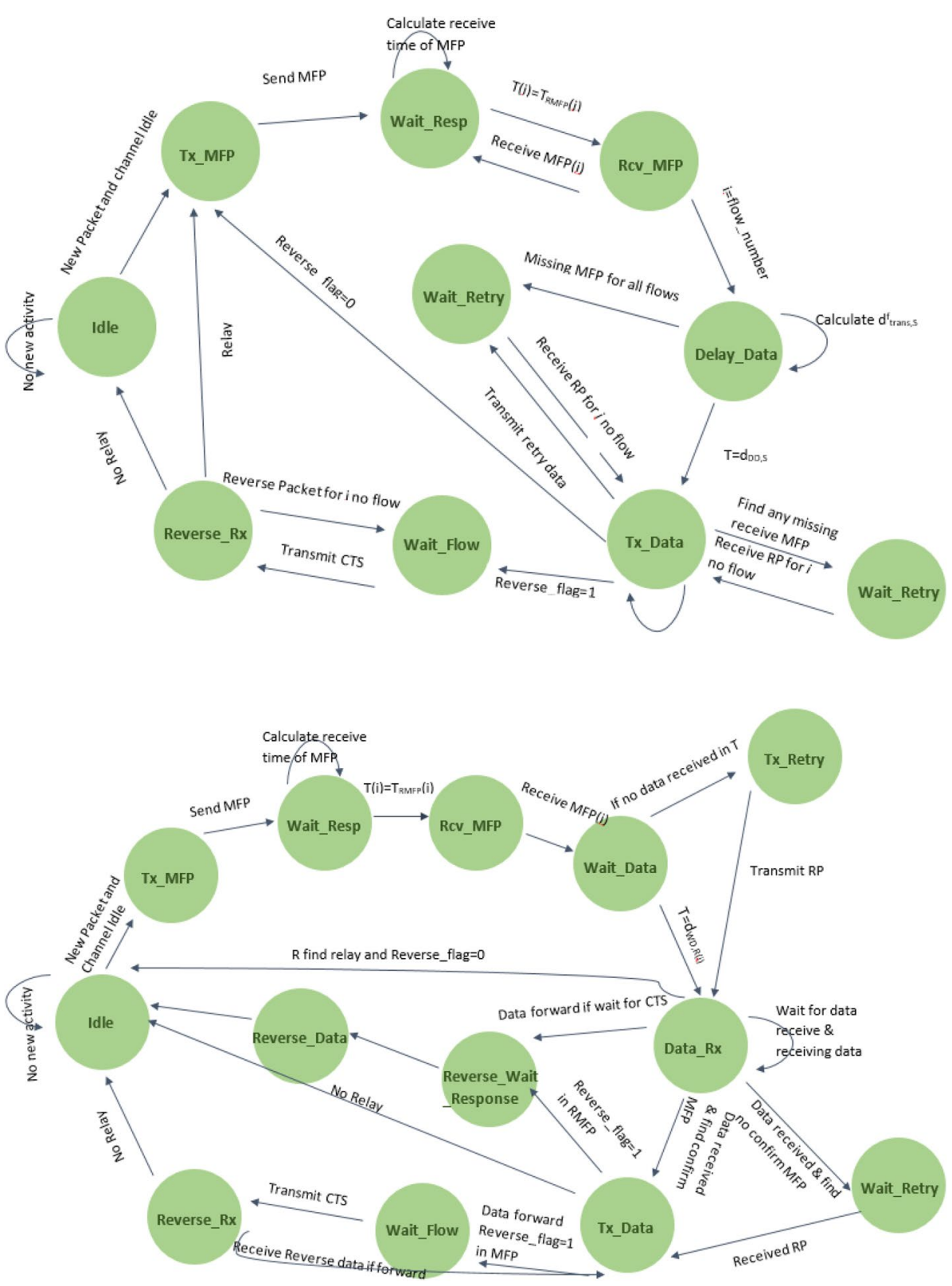


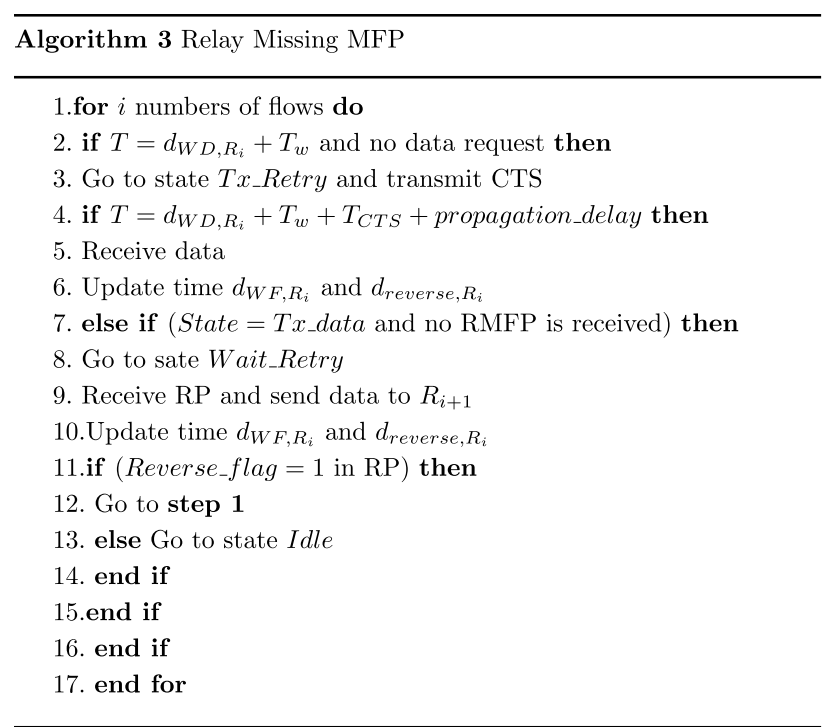

\section{State evolution process for sender node}

In the proposed protocol, the state progress outline of a sender node is deciphered in Fig. 8. This outline depicted the lead of a sender how it transmits multi-stream multijump packets (Fig. 9).

\subsection{TX_MFP state}

It is referenced that, the sender sends MFP to the hand-off hubs in TX_MFP state over multi-streams. Sender enters to the Wait_Resp condition in the wake of sending MFP bundle and the sender hub gauges $T_{R M F P}^{i}$ the getting time of MFP. The sender enters to the RCV_MFP state, if time is identical to the hour of getting of a MFP for $i$ number stream. The sender enters to Wait_Resp state, in the wake of getting MFP for $i$ number stream. In Wait_Resp express, the sender anticipates for the holding up time of next getting MFP $T_{R M F P}^{i+1}$. Afterward, it moved to $R C v \_M F P$ state again for accepting MFP of $i+1$ number stream at the hour of getting MFP. On the off chance that the quantity of stream $i$ is not exactly the stream development number the exchanging of states Wait_Resp and Rcv_MFP proceeds for multi-stream information transmission.

\subsection{Delay_Data state}

To transmit data group for various streams $i$ for sender $S$, learns $d_{\text {trans,S }}^{i}$ in Delay_Data state and sender center point holds on for $d_{D D}$ defer data time. A sender can wander out to two particular states from Delay_Data according to the cases. The sender moves to Tx_Data state if gets all tantamount response MFP by and large enters to Wait_Retry condition.

\subsection{Tx_Data state}

In Tx_Data state, three circumstances can occur. Case (1) If the sender doesn't miss MFP for any stream and reverse_flag $=1$ in affirm MFP, the sender goes to Wait_Flow state for starting information transmission over turn around stream. Case (2) If the sender finds any missing affirm MFP for any stream and reverse_flag $=1$, the sender goes to Wait_Retry state. Case (3) If reverse_flag $=0$ is distinguished by the sender, perceives no information transmission over turn around stream heading and moves to Idle state.

\subsubsection{Reverse data transmission}

On the off chance that (1) The sender delays for $d_{W F, S}^{i}$ (sit tight period for hand-off I in Wait_Flow state) period in the state Wait_Flow so as to dodge impact if doesn't slip MFP for streams and reverse_flag is set. Later on, sender transmits CTS bundle after this timeframe and enters to Reverse_Rx condition. Sender drives to the state Wait_Flow some other time and sits tight for $d_{\text {trans, }}^{i+1}$ time for getting opposite information transmission in the wake of accepting converse information parcels over next stream $i+1$. As the information transmission is finished, the sender node selects any one of these two states Wait_Flow and Reverse_Rx for all $f$ number of streams.

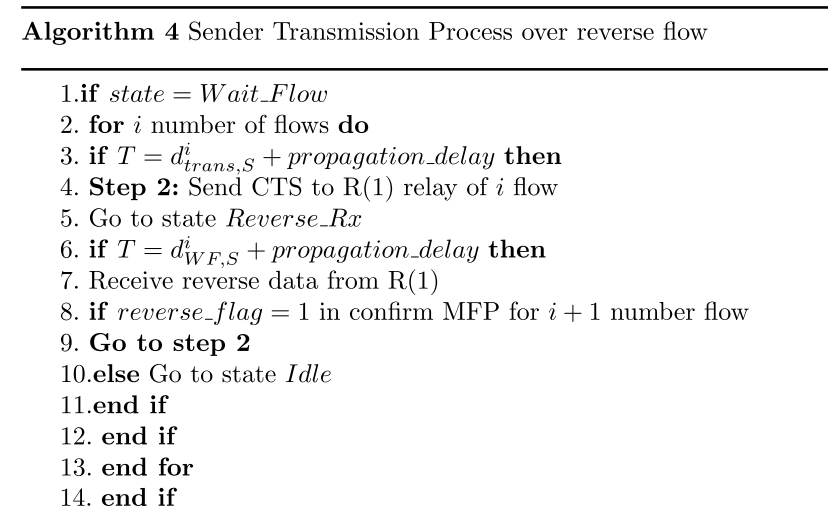

\subsubsection{Reverse_Rx state}

In case (3) In state Reverse_Rx the node gets information over invert flow heading. On the off chance that the sender node has no information to relay, it enters to state Idle; generally, the sender goes to state Tx_MRP. 
Algorithm 4 depicts the reverse flow transmission procedure of sender node $S$.

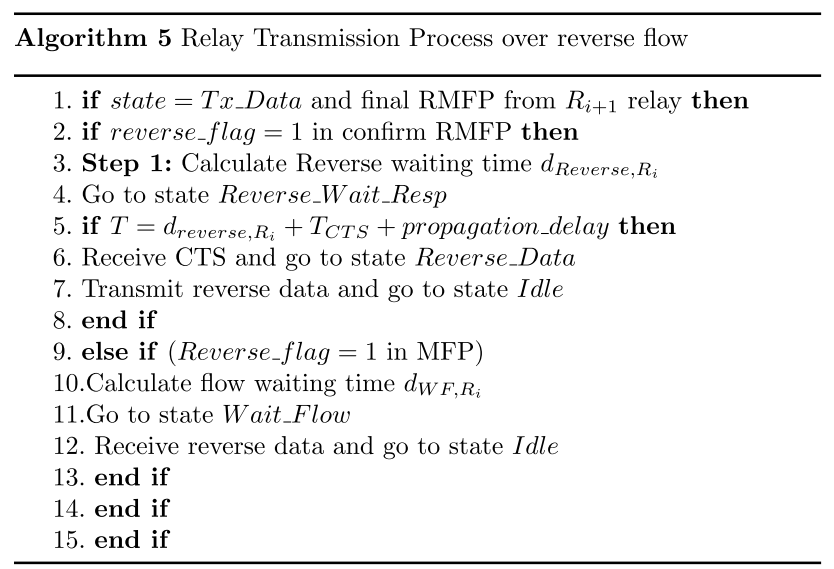

\section{Results and discussion}

The performance of proposed protocol is examined using a renowned simulation tool MATLAB with the parameter of energy, throughput and latency. Finally, in order to investigate the efficiency of the proposed BMFMAC protocol, a performance comparison between BMFMAC protocol and CMRT protocols is carried out.

\subsection{Analytical analysis}

In a multi-hop topology, 36 static nodes is considered for our scientific examination set in a area of dimension $5000 \times 5000 \mathrm{~m}^{2}$ illustrated in Fig. 10. The transmission extent of hub in separation is $1500 \mathrm{~m}$ while two hubs are set $1000 \mathrm{~m}$ separation in matrix dividing. It is understood that every hub has a comparative transmission control. In Fig. 10, the majority of the hubs are relied upon to include absolutely eight neighbors inside its range which is determined by the dabbed circle. The acoustic channel is dared to be blunder inclined while the normal transmitting and accepting force is $2 \mathrm{~W}$ and $20 \mathrm{~mW}$ for the acoustic handset.

\subsection{Experiment setup}

The network parameters and packet criteria for our experiment design have been fixed for BMF-MAC which is presented in Tables 1 and 2 respectively. The size of data packet is fixed 1200 bits as in CMRT protocol. The size of control packet MFP is 128 bits, which is one byte longer than that of CMRT protocol.

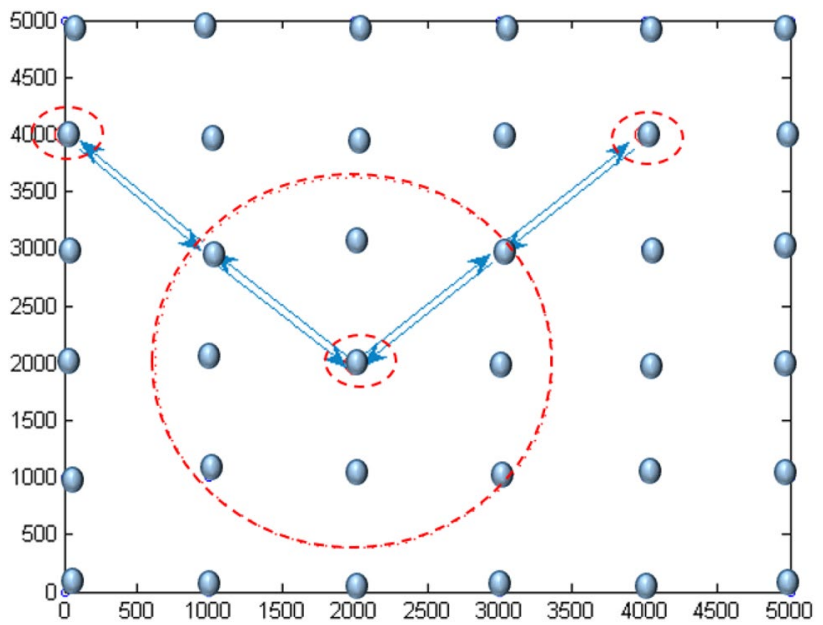

Fig. 10 The network topology for analysis

\subsection{Results and discussions}

In this subsection, the exhibition of the proposed BMF$M A C$ convention is investigated. In the new form of BMF convention which is known as BMF-M where just multistream data communication is taken care of. In fact, BMF-R anticipates multi-stream two-way data sending. Various outcomes are concentrated by three distinctive execution measurements: inertness, vitality productivity and throughput. Execution of both adaptation BMF-MAC convention is contrasted and the current CMRT convention.

\subsubsection{Throughput}

In this subsection, the performance of throughput of BMFMAC protocol is evaluated. Performance of throughput is

Table 1 Systems factors

\begin{tabular}{ll}
\hline Factors & Value \\
\hline Acoustic dissemination speed & $1500 \mathrm{~m} / \mathrm{s}$ \\
Sending rate & $9600 \mathrm{bps}$ \\
Buffer size $\left(N_{\max }\right)$ & 300 packets \\
Counter of minimum back-off & 1 \\
Counter of maximum back-off $\left(B_{\max }\right)$ & 64 \\
Bit rate & $1200 \mathrm{bps}$ \\
Tx power & $2 \mathrm{~W}$ \\
Rx power & $20 \mathrm{~mW}$ \\
Idle power & $0.8 \mathrm{~mW}$ \\
\hline
\end{tabular}

Table 2 Packet parameters 
studied with a view to various offered loads, numerous distances, flows, different network areas, number of reverse packets and BERs.

6.3.1.1 Effects of offered loads Figure 11 demonstrates that the information throughput of proposed convention and CMRT convention in various offered loads with BER of $10^{-3}$. The $x$-hub demonstrates the offered burden while the $y$-hub demonstrates the throughput as far as bit every second (bps). It is seen in all offered burden conditions that BMF-MAC demonstrates the best execution with respect to throughput. Additionally, the framework throughput demonstrates the information bundles which is gotten by both hand-off and last goal hubs effectively.

The framework throughput demonstrates the general channel usage by utilizing the MAC convention correspondingly. In this way, it is indistinguishable from standardized throughput per hub. Subsequently, it very well may be reasoned that, as far as channel usage, BMF-MAC outperforms other option. The result exhibits CMRT is inefficient, in light of the way that sender transmits data to beneficiary with per-round channel reservation over a singular stream, when the engendering deferral is high which encounters under-utilization of the channel.In any case, channel booking system permits lone sender to send data bundle with per round of channel booking to different hubs of a few streams. So as to improve channel work, it can lessen the hard and fast channel reservation overhead inconceivably. As needs be, BMF-MAC has favored data throughput over CMRT. Figure 11 exposes that, for high traffic stack 3 parcels/s, BMF-M can attain most significant addition of data throughput about $39.2 \%$ higher appeared differently in relation to CMRT convention similarly as BMF-R can attain the most essential augmentation of data throughput around $67.5 \%$ higher diverged from CMRT convention. BMF-M convention can attain throughput around $20 \%$ in low rush hour gridlock load 0.5 where BMF-R attain $46.7 \%$ higher than that of CMRT. More or less, BMF-MAC convention beats CMRT convention concerning data throughput in factor traffic loads.

6.3.1.2 Effects of inter nodal distance In the assortment of bury nodal partitions with BER of $10^{-3}$ the throughput of proposed convention and CMRT convention is delineated in Fig. 12 where offered burden is found to 0.8 parcels/s. The x-pivot exhibits the partitions however the $y$-hub shows the throughput to the extent bit every second (bps). It is broke down that the presentation of all of the conventions in regards to throughput spoils with the extending of the between nodal separation. This is a direct result of the climbing of the detachment related correspondence overhead. In light of deferred expansion postpone the clamoring length close by the handshak-

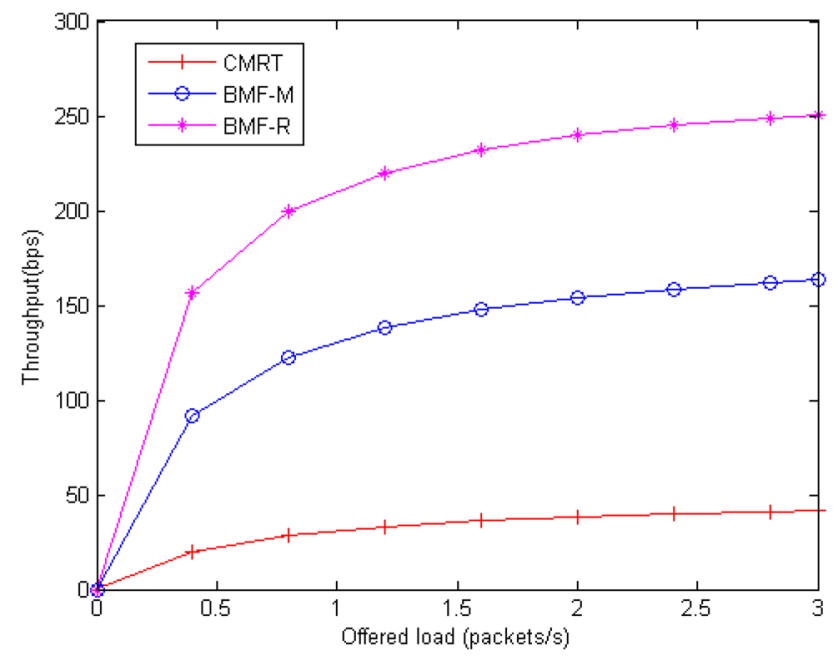

Fig. 11 Throughput versus offered load for CMRT protocol and the proposed BMF protocol

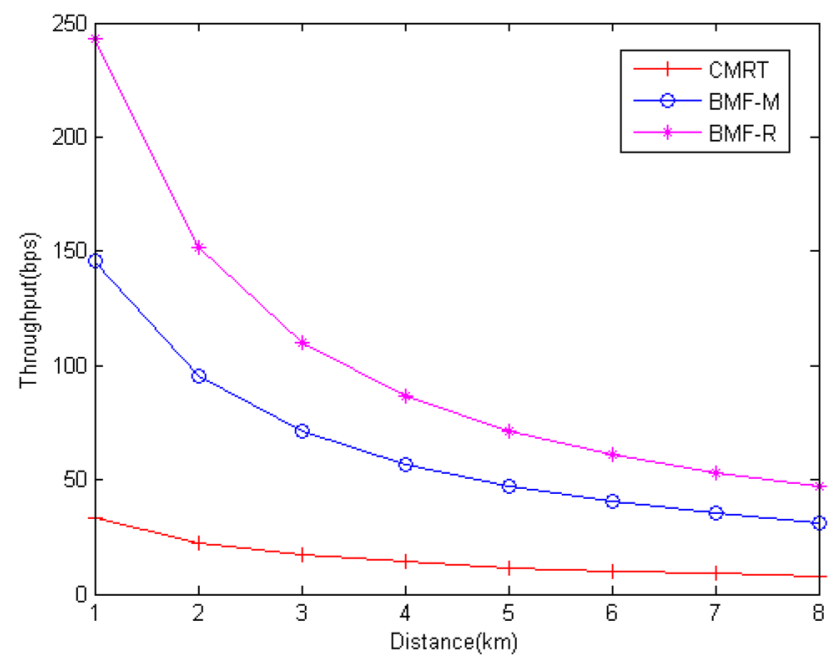

Fig. 12 Throughput versus distance for CMRT protocol and the proposed BMF protocol

ing time raises as the separation expanded. Thusly, with the extending of the widely inclusive involved term the Silence state length enhances. This makes a hub have less opportunity to trade control parcels.It is seen that, BMFMAC can give improved result appearing differently in relation to CMRT to manage variable traffic plans in multijump submerged sensor systems. For more diminutive bury nodal separation $1 \mathrm{~km}$, the throughput of BMF-R and BMF-M conventions can attain around $83 \%$ and $45 \%$ higher appeared differently in relation to CMRT convention separately tended to from Fig. 12 . On the off chance that there ought to be an event of medium bury nodal expel $4 \mathrm{~km}, \mathrm{BMF}-\mathrm{R}$ and BMF-M MAC convention can attain 
throughput around $27 \%$ and $17 \%$ more unmistakable than that of CMRT convention exclusively.

6.3.1.3 Effects of number of flows Advancement of different streams throughput of proposed convention and CMRT convention is spoken to in Fig. 13. For BER $10^{-3}$ and offered load 3.2 bundles/s, the $x$-hub shows the amount of streams while the $y$-pivot exhibits the throughput to the extent bit every second (bps). Figure 13 depicts the execution of both CMRT and BMF-MAC conventions in regards to throughput spoils with the growing of the amount of streams. Furthermore, different streams cause the traffic plan progressively erratic and hard to arrangement with by MAC show in submerged sensor organ-

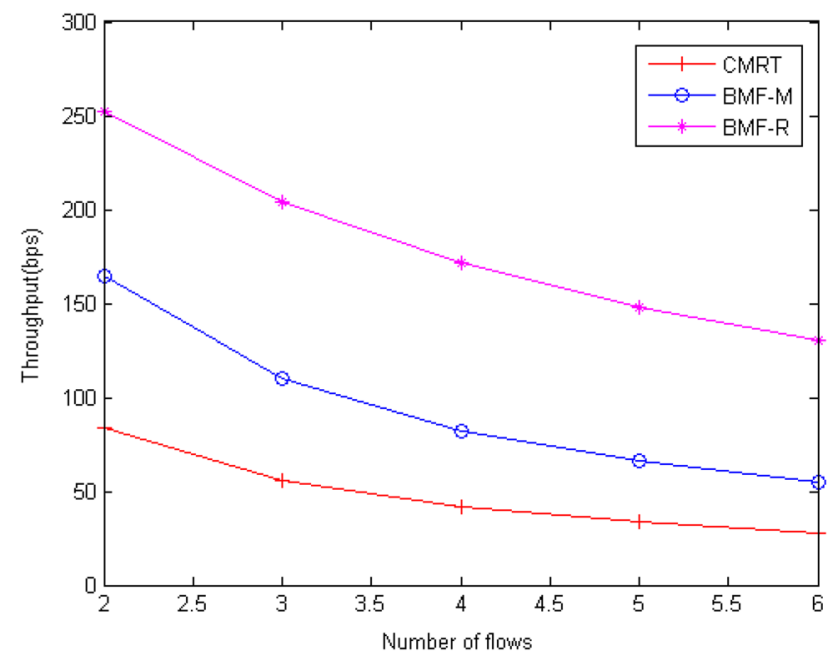

Fig. 13 Throughput versus no. of flows for CMRT protocol and the proposed BMF protocol

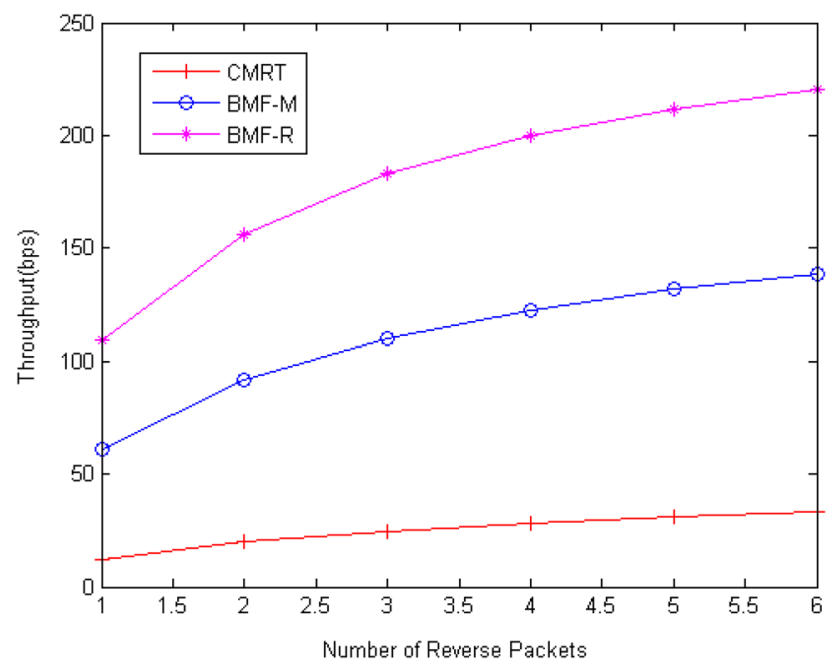

Fig. 14 Throughput versus no. of reverse packets for CMRT protocol and the proposed BMF protocol ize circumstance. From Fig. 13, it is seen that in twofold stream data transmission, the throughput of BMF-R and BMF-M conventions can attain around $70.1 \%$ and $40.1 \%$ higher stood out from CMRT convention independently.

6.3.1.4 Effects of number of reverse packets The $x$-axis of Fig. 14 demonstrates the quantity of reverse packets though the $y$-axis demonstrates the throughput regarding bit per second (bps). It is exhibited that the execution of both rendition of the BMF-MAC protocols as far as throughput raises with the expansion of the quantity of invert packets. BMF-MAC acquaints four sorts of states with handle invert packets. Along these lines, information transmission over multi-flow receiving with reverse information transmission method can happen with no impact. This makes a node have critical chance to trade bidirectional packets over multi-flows in less time prerequisite. From Fig. 14, it is recognized that while invert packet number is one, the throughput of BMF-R and BMF-M protocols can accomplish around $39 \%$ and $17 \%$ higher contrasted with CMRT protocol separately. In the event that number of invert information packets is increment to 6 , BMR-R can accomplish the most noteworthy accomplishment of throughput around $78 \%$ high contrasted with CMRT though BMF-M picks up the greatest increment of throughput around $46 \%$ extensive contrasted with CMRT. Likewise, BMF-MAC outperforms CMRT with the rising number of invert packets.

6.3.1.5 Effects of number of nodes For different number of nodes, Fig. 15 shows the throughput for 0.4 packets/s offered load. The $x$-axis shows number of nodes whereas the $y$-axis shows the throughput in terms of bps. As the number of nodes increase, the number of source nodes increase as well. Therefore, more multi-flow construc-

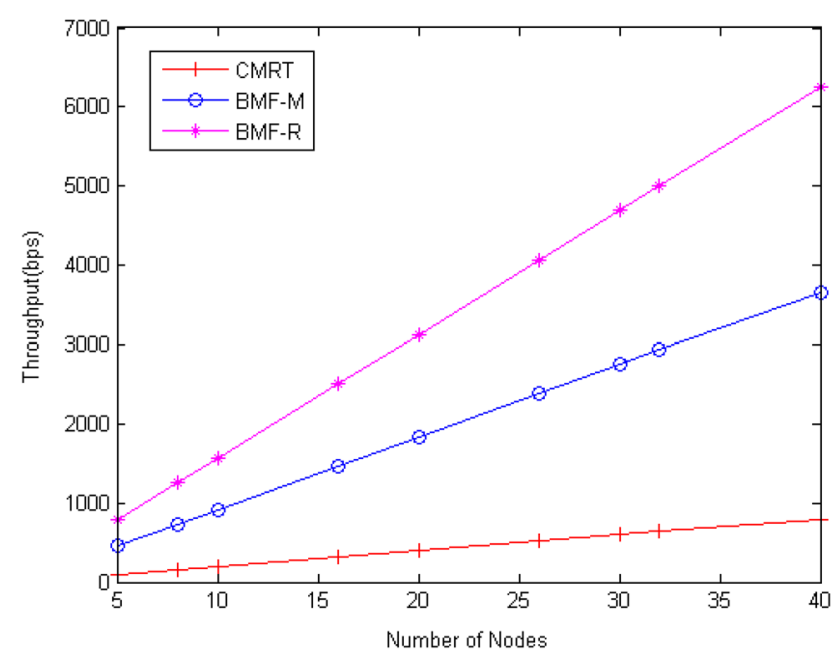

Fig. 15 Throughput versus no. of nodes for CMRT protocol and the proposed BMF protocol 
tions can take place which facilitates BMF-MAC protocol to deliver more data packets which results improvement of throughput. More specifically, for number of nodes 5, BMF-M MAC protocol can attain throughput around $5.6 \%$ higher compared to CMRT. On the other hand, BMF-R gains throughput around $9.6 \%$ higher compared to CMRT protocol. When the number of node is improved to 40, BMF-M MAC achieves the greatest improvement of throughput around $38.2 \%$ higher and BMF-R can gain $74.2 \%$ more than that of CMRT protocol. That reveals BMF-MAC protocol is more throughput efficient for large area network. The above mentioned information indicates that, BMF-MAC outperforms CMRT with respect to throughput with the increase of network size.

6.3.1.6 Effects of BER For different number of BERs, Fig. 16 interprets the data throughput with offered load 0.8 packets/s. Here, $x$-axis shows the BER whereas the $y$-axis shows the throughput in terms of bit per second. Different lines present the results collected with different protocols: BMF-MAC, RMAC LO-MAC. Throughput decreases when the bit error rate increases from 0 to 1 . It is observed that, the bidirectional multi flow data transmission technique in BMF-MAC significantly contributes to the improvement of throughput with the decrease of BER.

\subsubsection{Latency}

In this subsection, the performance of end to end delivery latency of BMF-MAC protocol is evaluated. Performance of latency is studied as indicated by various number of hops, numerous distances, flows, and different bit error rates (BERs).

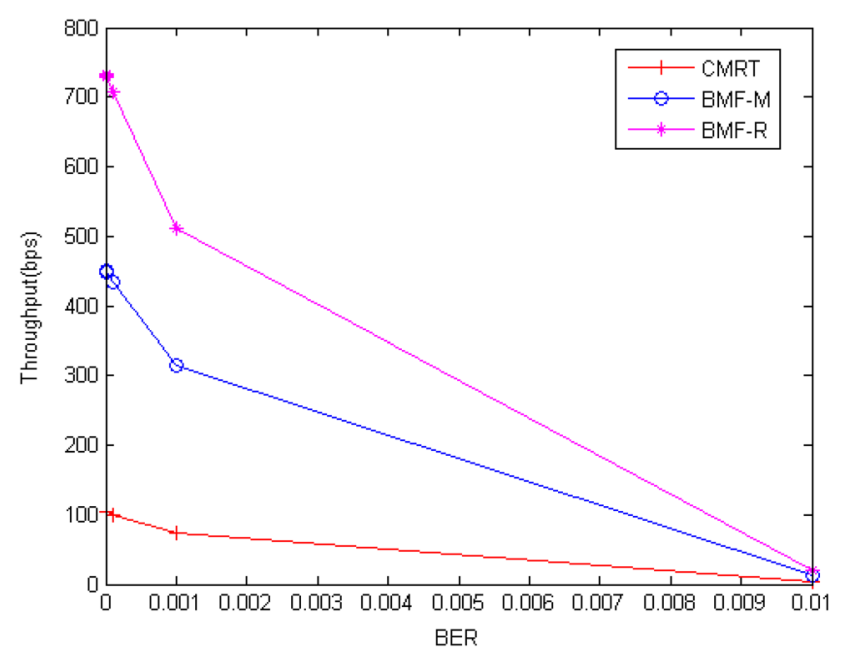

Fig. 16 Throughput versus BER for CMRT protocol and the proposed BMF protocol
6.3.2.1 Effects of number of hops In Fig. 17 End to end packet delay is introduced of proposed protocol and CMRT protocol in the variety of number of hops with BER of $10^{-3}$. The $x$-pivot exhibits the amount of bounces while the $y$-hub shows the idleness to the extent second (s). Particular lines present the results accumulated with different conventions: BMF-M MAC, BMF-R MAC and CMRT. As can be seen from the figure, inactivity increases when the amount of jumps increases from 1 to 10 . Regardless, bundles experience longer deferral while using CMRT strategy stood out from the BMF-MAC plot with the development of number of jumps. Even more effectively, BMF-M MAC convention furnishes $25 \%$ less bundle deferral stood out

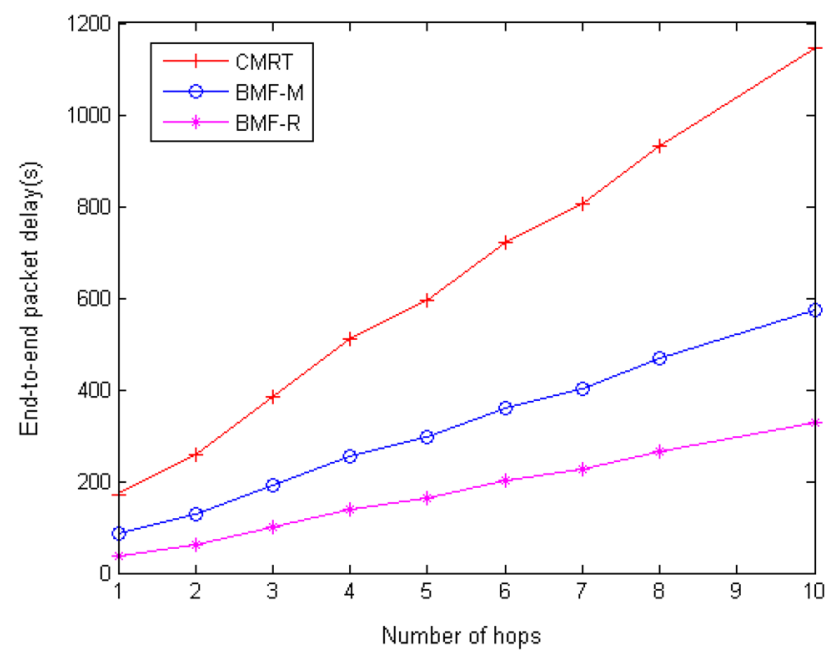

Fig. 17 Packet delay versus no. of hops comparisons of proposed and CMRT protocol

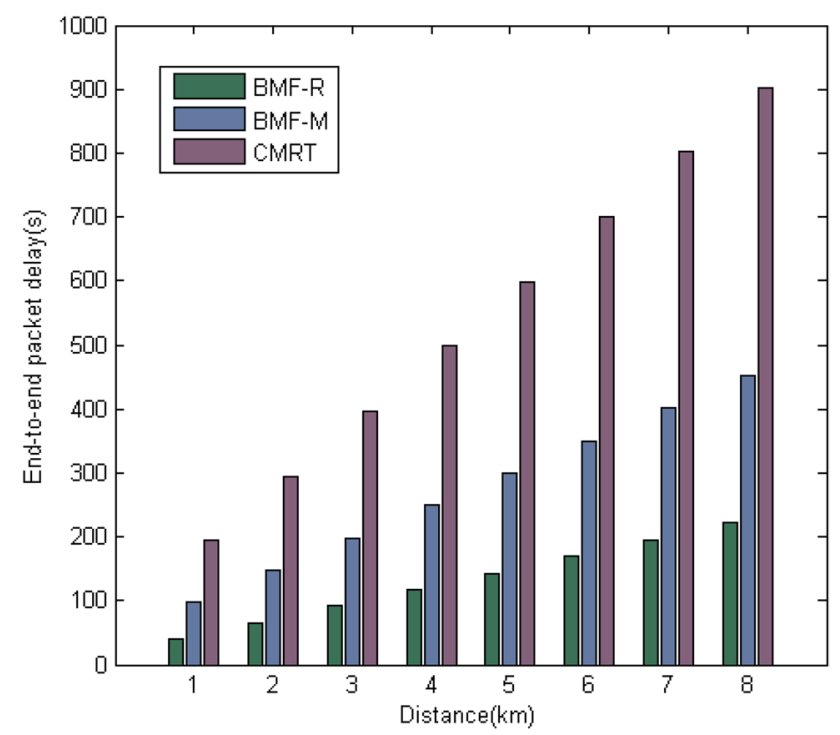

Fig. 18 Packet delay versus distance for CMRT protocol and the proposed BMF protocol 
from CMRT if there ought to emerge an event of medium number of 5 jumps, where inertness become $50 \%$ lower with a bounce tally of 10 . Of course, the BMF-R MAC convention gives $35 \%$ less bundle postponement appeared differently in relation to CMRT convention, in medium number of jump 5. Besides, inertness decline around $72 \%$ lower in high number of jump number 10 . Additionally likewise with the growing of number of bounces, more bundles can be passed on over different streams in a comparable time need by the BMF-MAC convention. Moreover, during the time spent extending of the bounce number, BMF-MAC can move progressively bidirectional bundles backward stream. Along these lines, it is seen that, the BMF-MAC convention outmaneuvers the current CMRT convention to the extent dormancy with the development of number of jumps.

6.3.2.2 Effects of inter nodal distance In Fig. 18 throughput of CMRT protocol and proposed protocol with BER of $10^{-3}$ in the different number of reverse packets is illustrated. The $x$-axis shows the number of reverse packets whereas the $y$-axis shows the throughput in terms of bit per second (bps).

6.3.2.3 Effects of number of flows With the expanding of inter nodal distance of node, end to end delay of proposed protocol and CMRT protocol in Fig. 18 is illustrated while BER is set to $10^{-3}$. As the separation builds, the bustling length alongside the packet exchange time raises due to delayed propagation delay. In this way, with the expanding of the all-encompassing duration the Wait_Flow state length grows. In order to transmit bidirectional packets,

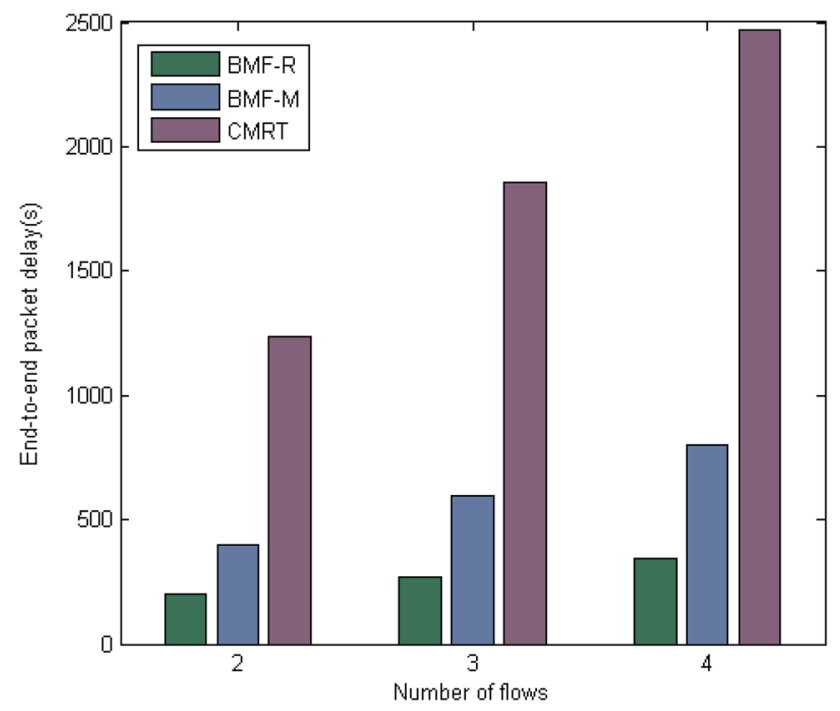

Fig. 19 Packet delay versus no. of flows for CMRT protocol and the proposed BMF protocol a node requires more opportunity in multi-flow situation. When the length is $1 \mathrm{~km}$, the proposed protocol gives $10 \%$ less packet delay contrasted with CMRT protocol illustrated in Fig. 18. With the increment of the distance to $4 \mathrm{~km}$, proposed protocol gives $25 \%$ less latency than CMRT. Proposed convention can give $45 \%$ less inactivity contrasted with the CMRT convention when the separation is expanded to $8 \mathrm{~km}$. Straightway, BMF-R MAC convention increments $15.2 \%$ less bundle postponement appeared differently in relation to the CMRT convention for bury nodal separation $1 \mathrm{~km}$. For the separation $4 \mathrm{~km}$, BMF-R MAC convention gives inertness around 39.5\% lower than that of CMRT. The proposed convention gives 69\% less inactivity contrasted and CMRT with the expansion of the separation $8 \mathrm{~km}$. In this manner, it is seen that, BMF-MAC can give a progressively ideal result to manage

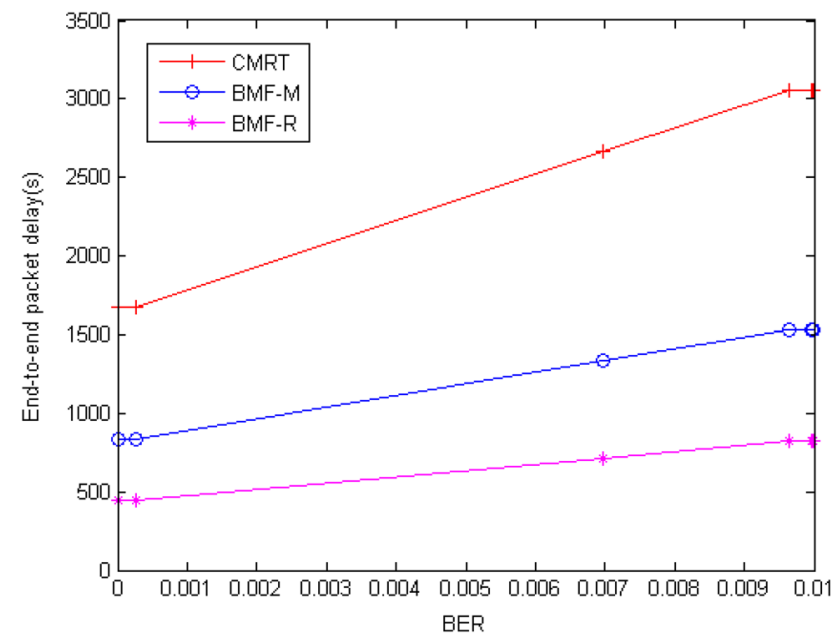

Fig. 20 Packet delay versus BER for CMRT protocol and the proposed BMF protocol

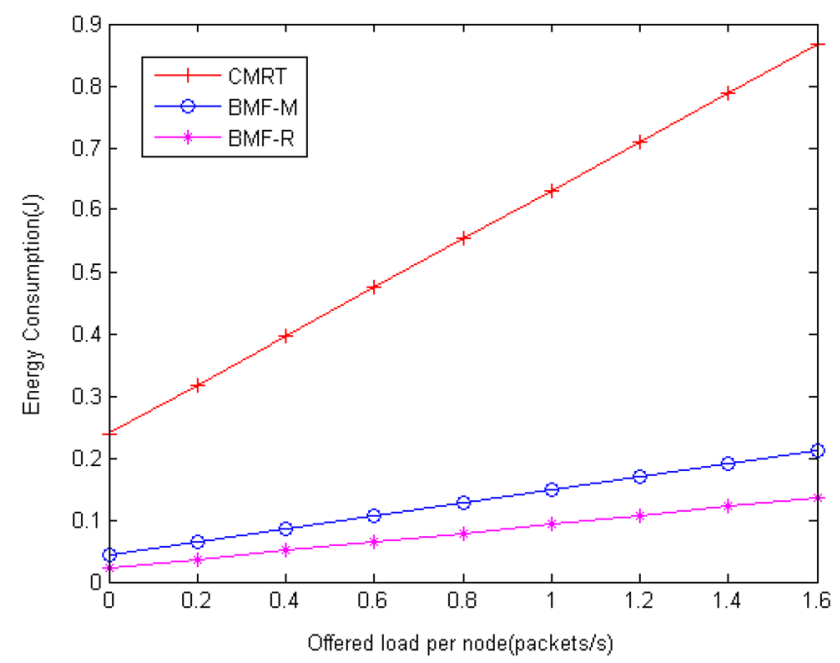

Fig. 21 Energy consumption versus offered load for CMRT protocol and the proposed BMF protocol 
variable traffic plans in multi-bounce submerged sensor system diverging from CMRT.

With the extension of number of streams the start to finish postponement of proposed convention and existing CMRT convention is illustrated in Fig. 19. For BER $10^{-3}$, the $x$-pivot shows the amount of streams while the $y$-hub exhibits the start to finish delay similar to second. Figure 19 outlines the execution of both CMRT and BMF-MAC convention to the extent start to finish defer diminishes with the extending of the amount of streams. Proposed convention gives $34 \%$ less bundle deferral contrasted and CMRT outlined in Fig. 19 where proposewd convention gives $76 \%$ less inactivity high number of stream number 4. Likewise, data transmission over twofold streams, BMF-R MAC convention increments $40 \%$ less parcel postponement appeared differently in relation to CMRT convention where inactivity become $88 \%$ less with high number of stream number 4.

6.3.2.4 Effects of BER For different number of BERs, Fig. 20 interprets the end-to-end delay. Here, $x$-axis shows the $B E R$ whereas the $y$-axis shows the end-to-end delay in terms of bit per second. Different lines present the results collected with different protocols: BMF-MAC, CMRT. Throughput decreases when the bit error rate increases from 0 to 1 . The six new states in BMF-MAC significantly contributes to the improvement of latency with the increase of BER. It is observed that, the bidirectional multi flow data transmission technique in BMF-MAC significantly contributes to the improvement of end-to-end delay with the decrease of BER.

\subsubsection{Energy consumption}

The performance of energy consumption of BMF-MAC protocol is evaluated in this subsection. According to inter nodal distance the performance of energy consumption is studied, different number of flows, various offered loads and different number of nodes (Fig. 21).

6.3.3.1 Effects of offered loads With the increase of offered load, energy consumption of proposed protocol and CMRT protocol is outlined in Fig. 22. The $x$-axis shows the offered loads whereas the $y$-axis shows the energy consumption in terms of joule (J). BMF-MAC reduces transmission time as it allows nodes to transmit simultaneously when there is multiple data to transmit over multi flow. Hence, it needs less energy than CMRT protocol in all traffic load scenario. Since BMF-MAC needs less control packets exchange than CMRT protocols it also requires less energy in all traffic load condition. Moreover, data packets can be delivered over regular and reverse flow direction without collusions using six different states in
BMF-MAC protocol. More specifically, it is seen that in low traffic load for offered load 0.2 packet/s, the energy consumption of proposed protocol can achieve around $28 \%$ less energy consumption contrasted with CMRT protocol.

6.3.3.2 Effects of number of nodes For various number of hubs Fig. 23 demonstrates the vitality utilization for offered load. The $x$-hub shows number of hubs though the $y$-pivot demonstrates the vitality utilization as far as joule. For number of hubs 20, BMF-M MAC convention can accomplish vitality utilization around $37.5 \%$ less contrasted with CMRT. Then again, BMF-R picks up vitality utilization around $49.2 \%$ less differentiated to the CMRT convention. At the point when the quantity of hub is

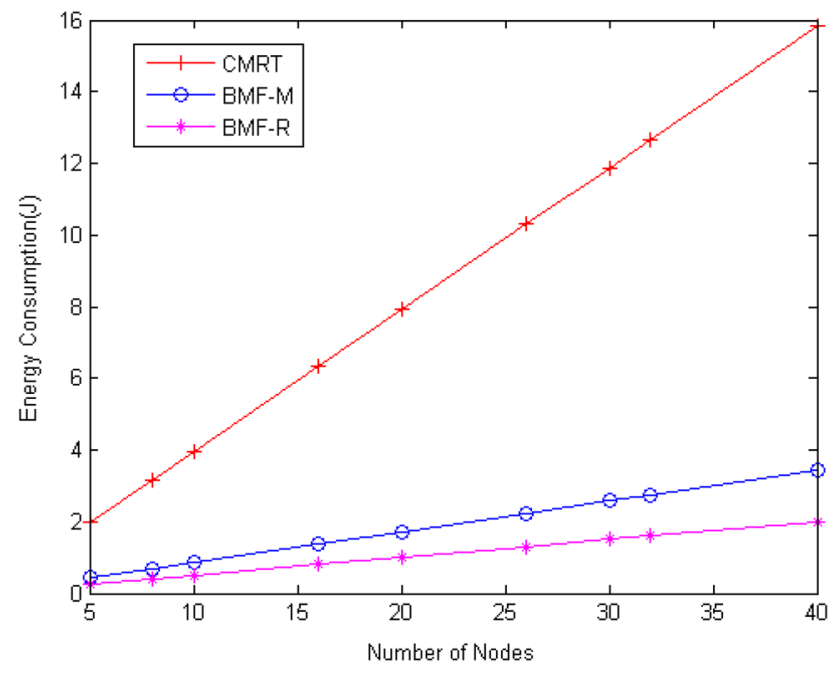

Fig. 22 Energy consumption versus no. of modes for CMRT proto$\mathrm{col}$ and the proposed BMF protocol

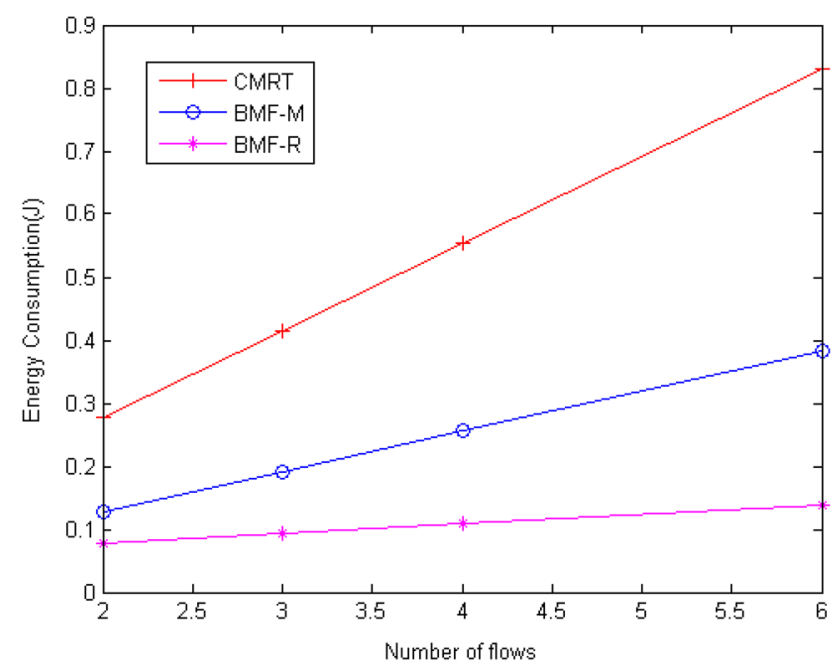

Fig. 23 Energy consumption versus no. of flows for CMRT protocol and the proposed BMF protocol 
expanded to 40, BMF-M MAC can accomplish the most noteworthy improvement of vitality utilization around $76 \%$ lower and BMF-R can increase $87.5 \%$ not as much as that of CMRT convention. That uncovers BMF-MAC convention is more vitality productive for enormous region arrange. The previously mentioned data shows that, BMFMAC outflanks CMRT concerning vitality utilization with the expansion of system size. As the quantity of source hubs increments With the expanding of the quantity of hubs. Hence, progressively bidirectional multi-stream developments can occur which encourages BMF-MAC convention to lessen control bundles overhead which results improvement of vitality utilization. Finally, we conclude that, the bidirectional multi-flow data transmission technique in BMF-MAC significantly contributes to the improvement of energy consumption for improved number of nodes.

6.3.3.3 Effects of number of flows In the assortment of entomb nodal divisions, vitality use of the proposed convention and the CMRT convention with a BER of $10^{-3}$ is found in Fig. 24. The $x$-hub shows the separation, while the $y$-pivot exhibits the vitality usage to the extent Joule $(J)$. It is demonstrated that the execution of all of the conventions to the extent vitality usage degrades with the extending of between nodal separation, as a result of the rising of the separation related correspondence overhead. As the separation improves, the clamoring term close by the bundle trade time increments because of a postponed engendering delay. Regardless, BMF-MAC can give improvement to manage variable traffic plans in multi-jump submerged sensor system standing out from CMRT.

Found in Fig. 24, with data transmission over twofold streams, the BMF-M MAC convention gives $18 \%$ less vitality usage appeared differently in relation to CMRT. During 4 streams, the BMF-M MAC convention can give vitality use around $49 \%$ under that of the CMRT convention. In addition, with data transmission over twofold streams, the BMF-R MAC convention increments $23 \%$ less vitality usage appeared differently in relation to the CMRT convention. Besides, for 4 streams, BMF-R MAC can get the degradation of vitality usage around $78 \%$ less appeared differently in relation to the CMRT convention.

6.3.3.4 Effects of inter nodal distance In the assortment of bury nodal separation, vitality usage of the proposed convention and the CMRT convention with a BER of $10^{-3}$ is found in Fig. 24. The x-pivot exhibits the partitions, while the $y$-hub shows the vitality usage to the extent Joule(J). It is shown that the execution of all of the shows similarly as vitality usage increases with the extending of the between nodal separation.

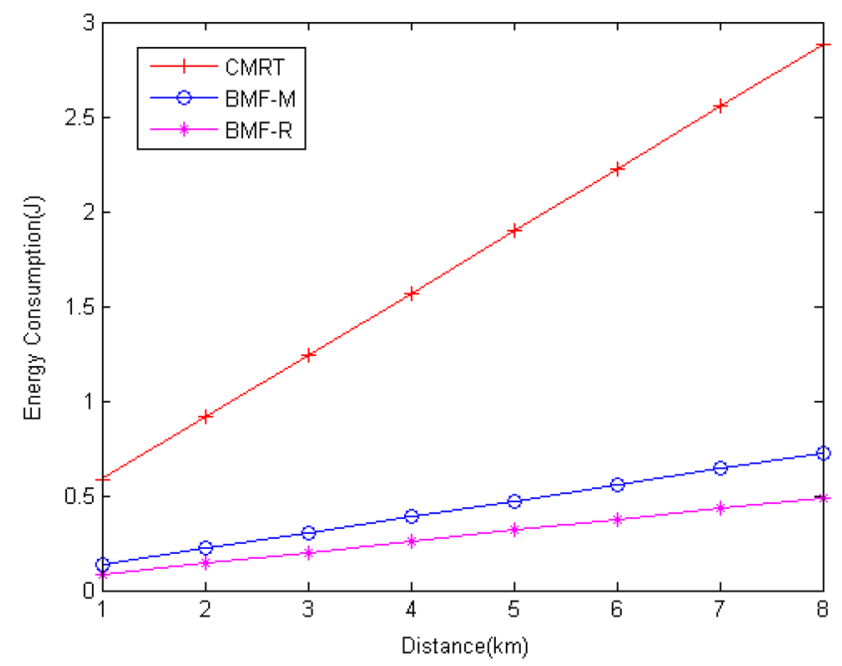

Fig. 24 Energy consumption versus distance for CMRT protocol and the proposed BMF protocol

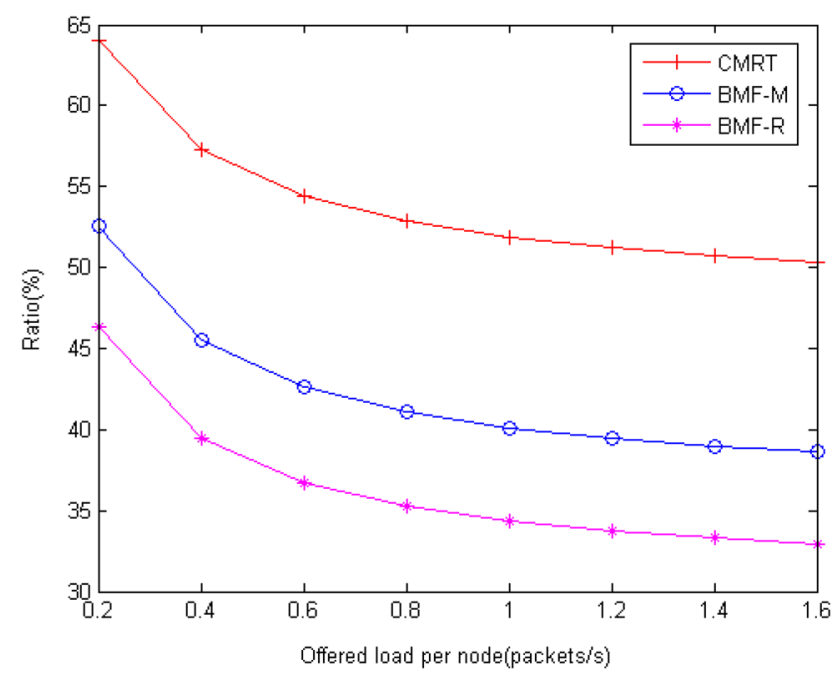

Fig. 25 Performance comparisons of BMF-MAC with CMRT in terms of control packet time

In particular, the proposed protocol gives $12.9 \%$ less energy utilization compared to the CMRT protocol, when the distance is $1 \mathrm{~km}$, as illustrated in Fig. 25 where the energy utilization decreases around $74.7 \%$ below when the distance is expanded to $6 \mathrm{~km}$. Straightway, the BMF-R MAC protocol increases $14 \%$ less energy utilization contrasted with the CMRT protocol in inter nodal distance $1 \mathrm{~km}$. For a distance of $6 \mathrm{~km}$, the BMF-R MAC protocol picks up the most elevated decrease of energy utilization around $76.3 \%$ lower than that of CMRT. Subsequently, with variable traffic designs in multi-hop underwater sensor networks, the proposed protocol can give better outcome contrasting with CMRT. 


\subsubsection{Control packet time}

Figure 25 shows that the control packet time of the proposed protocol and the CMRT protocol in different offered loads with a BER of $10^{-3}$. The $x$-axis shows the offered load, whereas the $y$-axis shows the control packet time in terms of seconds. In the BMF-MAC protocol, multiple control frames can be exchanged simultaneously; thus less control packet time is required. Furthermore, while more packets are generated, less control packets are required by the BMF-MAC protocol, thus less control packet time is needed. It is recognized that, in terms of control packet time, the proposed protocol exhibits the best performance in various offered load conditions.

\section{Conclusion}

In this paper, the execution assessment results, as far as energy utilization, packet latency and throughput of proposed protocol are explored. Additionally, to demonstrate the reasonableness of proposed convention, execution correlations of proposed protocol with that of CMRT protocol are completed. These results demonstrate that the proposed protocol performs better than the CMRT protocol regarding end to end delay, with the biggest enhancement of $88 \%$ in a huge number of streams. BMF$M A C$ saves more energy under factor traffic loads. If there should arise an occurrence of high traffic load, BMF-MAC can accomplish the most astounding expanding of information throughput around $67.5 \%$ higher than CMRT. By diminishing the end to end latency, the proposed MAC protocol performs better and has less energy utilization while expanding the throughput in UW-ASNs, under all traffic load cases. Along these lines, the proposed protocol outperforms the CMRT protocol to deal with variable traffic load designs.

Acknowledgements The authors would like to thank Shamim Ara Shawkat of University of Tennessee and Asad Hoque of East Tennessee State University for their helpful advice on preparing the preliminary version of this paper.

\section{Compliance with ethical standards}

Conflict of interest The authors declare that there is no conflict of interest.

\section{References}

1. Gkikopouli A, Nikolakopoulos G, Manesis S (2012) A survey on underwater wireless sensor networks and applications. In: 2012 20th Mediterranean conference on control \& automation (MED), pp 1147-1154. IEEE

2. Pompili D, Melodia T, Akyildiz IF (2006) Routing algorithms for delay-insensitive and delay-sensitive applications in underwater sensor networks. In: Proceedings of the 12th annual international conference on mobile computing and networking, $\mathrm{pp}$ 298-309. ACM

3. Liao W-H, Kuai S-C, Lin Y-C (2015) A receiver-initiated MAC protocol with packet train design for underwater acoustic sensor networks. Wirel Pers Commun 82(4):2155-2170

4. Freitag L, Grund M, Singh S, Partan J, Koski P, Ball K (2005) The WHOI micro-modem: an acoustic communications and navigation system for multiple platforms. In: Proceedings of OCEANS 2005 MTS/IEEE, pp 1086-1092. IEEE

5. Heidemann J, Ye W, Wills J, Syed A, Li Y (2006) Research challenges and applications for underwater sensor networking. In: IEEE wireless communications and networking conference, 2006, WCNC, vol 1, pp 228-235. IEEE

6. Chen K, Ma M, Cheng E, Yuan F, Wei S (2014) A survey on MAC protocols for underwater wireless sensor networks. IEEE Commun Surv Tutor 16(3):1433-1447

7. Rahman J, Alam MS, Shawkat SA, Hoque MA (2017) BMF-MAC: a bidirectional multi-flow MAC protocol for multihop underwater acoustic sensor networks. In: Proceedings of the international conference on underwater networks \& systems. ACM, p 13

8. Chirdchoo N, Soh W, Chua KC (2008a) RIPT: a receiver-initiated reservation-based protocol for underwater acoustic networks. IEEE J Sel Areas Commun 26(9):1744-1753

9. $\mathrm{Ng} \mathrm{H}-\mathrm{H}$, Soh W-S, Motani M (2013a) An underwater acoustic MAC protocol using reverse opportunistic packet appending. Comput Netw 57(14):2733-2751

10. Lee J-W, Cho H-S (2015) A hybrid sender-and receiver-initiated protocol scheme in underwater acoustic sensor networks. Sensors 15(11):28052-28069

11. Chirdchoo N, Soh W-S, Chua KC (2008b) MACA-MN: a MACAbased MAC protocol for underwater acoustic networks with packet train for multiple neighbors. In: VTC spring 2008-IEEE vehicular technology conference, pp 46-50. IEEE

12. Dou F, Peng Z (2015) On-demand pipelined MAC for multi-hop underwater wireless sensor networks. In: Proceedings of the 10th international conference on underwater networks \& systems, p 26. ACM

13. Lin W, Chen K (2016) MHM. Int J Distrib Sens Netw 2016:35

14. Rahman P, Karmaker A, Alam MS, Hoque MA, Lambert WL (2019) CUMAC-CAM: a channel allocation aware MAC protocol for addressing triple hidden terminal problems in multi-channel uwsns. SN Appl Sci 1(7):805

15. Rahman P, Alam MS, Shawkat SA, Hoque MA (2017) CUMACCAM: addressing triple hidden terminal problems for multichannel transmission in underwater sensor networks. In: Proceedings of the international conference on underwater networks \& systems, p 1. ACM

16. Karn P et al (1990) MACA-a new channel access method for packet radio. In: ARRL/CRRL amateur radio 9th computer networking conference, vol 140. Canada, London, pp 134-140

17. $\mathrm{Ng} \mathrm{H}-\mathrm{H}$, Soh W-S, Motani M (2008) MACA-U: a media access protocol MACfor underwater acoustic networks. In: IEEE GLOBECOM 2008 IEEE global telecommunications conference, pp 1-5. IEEE

18. Molins M, Stojanovic M (2007) Slotted FAMA: a MAC protocol for underwater acoustic networks. In: OCEANS 2006-Asia Pacific, pp 1-7. IEEE 
19. $\mathrm{Ng} \mathrm{H}-\mathrm{H}$, Soh W-S, Motani M (2013b) A bidirectional-concurrent MAC protocol with packet bursting for underwater acoustic networks. IEEE J Ocean Eng 38(3):547-565

20. Lee J-W, Cho H-S (2014) Cascading multi-hop reservation and transmission in underwater acoustic sensor networks. Sensors 14(10):18390-18409

21. Akyildiz IF, Pompili D, Melodia T (2004) Challenges for efficient communication in underwater acoustic sensor networks. ACM Sigbed Rev 1(2):3-8

22. Freitag L, Stojanovic M, Grund M, Singh S (2002) Acoustic communications for regional undersea observatories. In: Proceedings of oceanology international, pp 5-8

23. Akyildiz IF, Pompili D, Melodia T (2005) Underwater acoustic sensor networks: research challenges. Ad Hoc Netw 3(3):257-279

24. Akyildiz IF, Weilian S, Sankarasubramaniam Y, Cayirci E (2002) Wireless sensor networks: a survey. Comput Netw 38(4):393-422

25. Li N, Martínez J-F, Chaus JM, Eckert M (2016) A survey on underwater acoustic sensor network routing protocols. Sensors 16(3):414

26. Anwar A, Sridharan D (2015) A survey on routing protocols for wireless sensor networks in various environments. Int J Comput Appl 112(5):1-17
27. Han G, Jiang J, Bao N, Wan L, Guizani M (2015) Routing protocols for underwater wireless sensor networks. IEEE Commun Mag 53(11):72-78

Publisher's Note Springer Nature remains neutral with regard to jurisdictional claims in published maps and institutional affiliations. 